\title{
An existence proof for the gravitating BPS monopole *
}

\author{
Todd A. Oliynyk ${ }^{\dagger \ddagger}$ \\ Department of Mathematical and Statistical Sciences \\ University of Alberta \\ Edmonton AB T6G 2G1
}

\begin{abstract}
We prove the existence of the gravitating BPS monopole in EinsteinYang-Mills-Higgs (EYMH) theory. Existence is established using a Newtonian perturbation argument which shows that a Yang-Mills-Higgs BPS monopole solution can be be continued analytically in powers of $1 / c^{2}$ to an EYMH solution.
\end{abstract}

\section{Introduction}

In this paper we rigorously prove the existence of the gravitating Bogomol'nyiPrasad-Sommerfield (BPS) monopole which has been constructed numerically in [4]. We prove existence by using a Newtonian perturbation argument to show that the flat space Yang-Mills-Higgs (YMH) BPS monopole solution [14] can be continued analytically to a Einstein-Yang-Mills-Higgs (EYMH) solution which we refer to as the gravitating BPS monopole. The Newtonian perturbation argument in the form that is employed in this paper was developed by Lottermoser in [13] and subsequently used by Heilig to establish the existence of slowly rotating stars [9]. For an elegant alternate presentation of the Newtonian perturbation formalism using different but equivalent variables see [3].

The results of Heilig and of this paper show that the Newtonian perturbation method is a powerful method for obtaining existence theorems in general relativity for static or stationary matter models. In addition to establishing existence, the method also provides an analytic deformation from a Newtonian solution to its general relativistic counterpart. The deformation parameter is $1 / c^{2}$ where $c$ is the speed of light. So a Taylor expansion in $1 / c^{2}$ can be considered as a converging

*2000 Mathematics Subject Classification Primary 35Q75; Secondary 83C20 .

${ }^{\dagger}$ Present address: School of Mathematical Sciences, Monash University, VIC 3800 Australia

¥todd.oliynyk@sci.monash.edu.au 
post-Newtonian expansion. In this way, the Newtonian perturbation argument can be thought of as the inverse of the Newtonian limit where Newtonian solutions are obtained from general relativistic ones via the limit $1 / c^{2} \rightarrow 0$. An attractive feature of the method is that it produces solutions to the Einstein field equations where the matter fields are uniformly close to their corresponding Newtonian solutions. This means that the properties of the Newtonian solution pass directly to the corresponding relativistic solution.

In [11] it is shown how to formulate the Newtonian limit of the EYMH equations. The limiting equations have the important property that the Newtonian potential and the YMH fields decouple. Moreover, the static equations coincide with the static YMH equations on Minkowski space. Since the BPS monopole is a static solution to the YMH equations on Minkowski space, it can be interpreted as a solution of the Newtonian YMH equations. Although we use a different formalism from [11], the results are the same. We find that in the limit as $1 / c^{2} \rightarrow 0$, the YMH variables decouple from the Newtonian potential and also they satisfy the static YMH equations. This allows us to use the BPS monopole solution as the starting point for the perturbation argument. Also, the fact that the Newtonian potential decouples from the YMH variables in the limit $1 / c^{2} \rightarrow 0$ helps to make the perturbation argument relatively simple.

The paper is organized as follows: in section 2 we set up the field equations in a form suitable to use the Newtonian perturbation argument while in section 3 we review the theory of weighted Sobolev spaces which will be essential to our existence proof. The Banach spaces for our field variables (i.e. the Higgs field, gauge potential, and metric density) are set up in section 4 and then in section 6 the field equations are shown to be analytic on those spaces. Sections 78 contain the Newtonian perturbation argument. In these sections it is shown that BPS monopole solution can be continued analytically to a solution of the full EYMH equations.

\section{EYMH equations}

For indexing of tensors and related quantities Greek indices, $\alpha, \beta, \gamma$ etc., will always run from 0 to 4 while Roman indices, $i, j, k$ etc., will range from 1 to 3 . Partial derivatives will be denoted both by $\partial_{\alpha} u$ and $u_{, \alpha}$ while covariant derivatives will be denoted by $\nabla_{\alpha}$.

Let $g$ denote the Minkowski metric on $\mathbb{R}^{4}$. Fix a global coordinate system $\left(x^{0}, x^{1}, x^{2}, x^{3}\right)$ so that

$$
\underset{o}{g_{\alpha \beta}}=\operatorname{diag}\left(-\lambda^{-1}, 1,1,1\right) \quad \lambda:=\frac{1}{c^{2}}
$$


where $c$ is the speed of light. Define $\underset{o}{g \alpha \beta}$ by $\underset{o}{\left(g^{\alpha \beta}\right)}:=\underset{o}{\left(g_{\alpha \beta}\right)^{-1}}$ which gives

$$
g_{o}^{\alpha \beta}=\operatorname{diag}(-\lambda, 1,1,1) .
$$

Define the Minkowski metric density

$$
\underset{o}{\mathfrak{g}^{\alpha \beta}}:=\underset{o}{|g|^{\frac{1}{2}} g_{o}^{\alpha \beta}} \text { where } \underset{o}{|g|}:=\mid \operatorname{det} \underset{o}{\left(g_{\alpha \beta}\right) \mid} .
$$

Assume that $g_{\alpha \beta}$ is another Lorentzian metric defined on $\mathbb{R}^{4}$. Let $\left(g^{\alpha \beta}\right):=$ $\left(g_{\alpha \beta}\right)^{-1}$ and introduce the density

$$
\mathfrak{g}^{\alpha \beta}:=|g|^{\frac{1}{2}} g^{\alpha \beta} \quad \text { where } \quad|g|:=\left|\operatorname{det}\left(g_{\alpha \beta}\right)\right| .
$$

Following Lottermoser [13], we form the tensor density

$$
\mathfrak{U}^{\alpha \beta}:=\frac{1}{4 \lambda^{\frac{3}{2}}}\left(\mathfrak{g}^{\alpha \beta}-\underset{o}{\mathfrak{g}^{\alpha \beta}}\right)
$$

which will be taken as our primary gravitational variable. Observe that the metric $g^{\alpha \beta}$ can be recovered from $\mathfrak{U}^{\alpha \beta}$ by

$$
g^{\alpha \beta}=\frac{1}{\sqrt{|g|}} \mathfrak{g}^{\alpha \beta}
$$

where $\mathfrak{g}^{\alpha \beta}=\mathfrak{g}_{o}^{\alpha \beta}+4 \lambda^{\frac{3}{2}} \mathfrak{U}^{\alpha \beta}$ and $|g|=\left|\operatorname{det}\left(\mathfrak{g}^{\alpha \beta}\right)\right|$.

The Einstein equations can be written in terms of the density (2.5) as [13],

$$
4 \pi G|\mathfrak{d}| T^{\alpha \beta}=A^{\alpha \beta}+B^{\alpha \beta}+C^{\alpha \beta}+D^{\alpha \beta},
$$

where

$$
\begin{aligned}
& \underset{o}{\overline{\mathfrak{g}}^{\alpha \beta}}:=\sqrt{\lambda \mathfrak{g}_{o}}{ }^{\alpha \beta},
\end{aligned}
$$

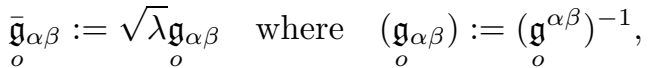

$$
\begin{aligned}
& \overline{\mathfrak{g}}^{\alpha \beta}:=\sqrt{\lambda} \mathfrak{g}^{\alpha \beta}=\overline{\mathfrak{g}}_{o}^{\alpha \beta}+4 \lambda^{2} \mathfrak{U}^{\alpha \beta}, \\
& \overline{\mathfrak{g}}_{\alpha \beta}:=\sqrt{\lambda} \mathfrak{g}_{\alpha \beta} \quad \text { where } \quad\left(\mathfrak{g}_{\alpha \beta}\right):=\left(\mathfrak{g}^{\alpha \beta}\right)^{-1}, \\
& \mathfrak{d}:=\lambda \operatorname{det}\left(\mathfrak{g}^{\alpha \beta}\right), \\
& A^{\alpha \beta}:=2\left(\frac{1}{2} \overline{\mathfrak{g}}_{\mu \nu} \overline{\mathfrak{g}}_{\gamma \rho}-\overline{\mathfrak{g}}_{\rho \mu} \overline{\mathfrak{g}}_{\gamma \nu}\right)\left(\overline{\mathfrak{g}}^{\alpha \kappa} \overline{\mathfrak{g}}^{\beta \sigma}-\frac{1}{2} \mathfrak{g}^{\alpha \beta} \overline{\mathfrak{g}}^{\kappa \sigma}\right) \mathfrak{U}^{\mu \nu}{ }_{, \kappa} \mathfrak{U}^{\gamma \rho}{ }_{, \sigma}, \\
& B^{\alpha \beta}:=4 \lambda \overline{\mathfrak{g}}_{\kappa \sigma}\left(2 \overline{\mathfrak{g}}^{\gamma(\alpha} \mathfrak{U}^{\beta) \sigma}{ }_{, \rho} \mathfrak{U}^{\kappa \rho}{ }_{, \gamma}-\frac{1}{2} \overline{\mathfrak{g}}^{\alpha \beta} \mathfrak{U}^{\kappa} \rho_{\gamma} \mathfrak{U}^{\sigma \gamma}{ }_{\rho}-\overline{\mathfrak{g}}^{\gamma \rho} \mathfrak{U}^{\alpha \kappa}{ }_{,} \mathfrak{U}^{\beta \sigma}{ }_{\rho}\right), \\
& C^{\alpha \beta}:=4 \lambda^{2}\left(\mathfrak{U}^{\alpha \beta}{ }_{, \kappa} \mathfrak{U}^{\kappa \rho}{ }_{, \rho}-\mathfrak{U}^{\alpha \kappa}{ }_{, \rho} \mathfrak{U}^{\beta \rho}{ }_{, \kappa}\right), \\
& D^{\alpha \beta}:=\overline{\mathfrak{g}}^{\mu \nu} \mathfrak{U}^{\alpha \beta}{ }_{, \mu \nu}+\overline{\mathfrak{g}}^{\alpha \beta} \mathfrak{U}^{\mu \nu}{ }_{, \mu \nu}-2 \mathfrak{U}^{\mu(\alpha}{ }_{, \mu \nu} \overline{\mathfrak{g}}^{\beta) \nu},
\end{aligned}
$$


and $T^{\alpha \beta}$ is the stress-energy tensor. As discussed in [9], any solution $\left(\lambda, \mathfrak{U}^{\alpha \beta}, T^{\alpha \beta}\right)$ of (2.7) for $\lambda>0$ is a solution of Einstein's equations displayed in units where $c=1 / \sqrt{\lambda}$. Following [9], we choose harmonic coordinates

$$
\nabla_{\alpha} \nabla^{\alpha} x^{\beta}=0, \quad \text { or equivalently } \mathfrak{U}_{, \beta}^{\alpha \beta}=0,
$$

which allows us to write the full Einstein field equations as

$$
\begin{gathered}
\mathfrak{U}^{\alpha \beta}{ }_{, \beta}=0, \\
4 \pi G|\mathfrak{d}| T^{\alpha \beta}=E^{\alpha \beta},
\end{gathered}
$$

where

$$
\begin{gathered}
E^{\alpha \beta}:={\underset{o}{o}}_{\overline{\mathfrak{g}}^{\mu \nu} \mathfrak{U}^{\alpha \beta}{ }_{, \mu \nu}+} 4 \lambda^{2}\left(\mathfrak{U}^{\mu \nu} \mathfrak{U}^{\alpha \beta}{ }_{, \mu \nu}+\mathfrak{U}^{\alpha \beta} \mathfrak{U}^{\mu \nu}{ }_{, \mu \nu}-2 \mathfrak{U}^{\mu(\alpha}{ }_{, \mu \nu} \mathfrak{U}^{\beta) \nu}\right) \\
+A^{\alpha \beta}+B^{\alpha \beta}+C^{\alpha \beta} .
\end{gathered}
$$

The equations (2.18) will be called the reduced field equations.

It is important to recognize that alone the reduced field equations (2.18) are not equivalent to the Einstein field equations (2.7). However, it is shown in [9] §6 that if $\nabla_{\beta} T^{\alpha \beta}=0$ and (2.18) can be solved and the stress-energy tensor $T^{\alpha \beta}$ satisfies certain conditions then the harmonic condition (2.17) will be automatically satisfied. In this case, a solution to (2.18) will actually be a solution to the full Einstein equation (2.7).

We will let $A=A_{\alpha} d x^{\alpha}$ denote the $S U(2)$-gauge potential and $\Phi$ the Higgs field. The $\mathrm{SU}(2)$ Yang-Mills-Higgs equations are

$$
\begin{gathered}
g^{\nu \alpha} D_{\nu}^{A} F_{\alpha \beta}^{A}=\left[\Phi, D_{\beta}^{A} \Phi\right], \\
g^{\nu \alpha} D_{\nu}^{A} D_{\alpha}^{A} \Phi=0,
\end{gathered}
$$

where

$$
D_{\alpha}^{A}(\cdot):=\nabla_{\alpha}(\cdot)+\left[A_{\alpha}, \cdot\right]
$$

is the gauge covariant derivative on gauge-scalars and

$$
F_{\alpha \beta}^{A}:=\partial_{\alpha} A_{\beta}-\partial_{\beta} A_{\alpha}+\left[A_{\alpha}, A_{\beta}\right]
$$

is the gauge field. For later use we define

$$
D_{\alpha}^{A}(\cdot):=\partial_{\alpha}(\cdot)+\left[A_{\alpha}, \cdot\right]
$$

which is the gauge covariant derivative on Minkowski space.

Multiplying (2.20) and (2.21) by $\sqrt{\lambda|g|}$ we find that

$$
\begin{gathered}
\overline{\mathfrak{g}}^{\alpha \nu}\left(F_{\alpha \beta, \nu}-\Gamma_{\alpha \nu}^{\mu} F_{\mu \beta}-\Gamma_{\beta \nu}^{\mu} F_{\alpha \mu}+\left[A_{\nu}, F_{\alpha \beta}\right]\right)-\sqrt{\mathfrak{d}}\left[\Phi, D_{\beta} \Phi\right]=0, \\
\overline{\mathfrak{g}}^{\alpha \beta}\left(\partial_{\alpha} D_{\beta} \Phi-\Gamma_{\alpha \beta}^{\mu} D_{\mu}^{A} \Phi+\left[A_{\alpha}, D_{\beta} \Phi\right]\right)=0,
\end{gathered}
$$


where the Christoffel $\Gamma_{\beta \gamma}^{\alpha}$ symbols are given by

$$
\Gamma_{\beta \gamma}^{\alpha}=\overline{\mathfrak{g}}^{\alpha \mu}\left(2 \overline{\mathfrak{g}}_{\beta \sigma} \overline{\mathfrak{g}}_{\gamma \tau}-\overline{\mathfrak{g}}_{\beta \gamma} \overline{\mathfrak{g}}_{\sigma \tau}\right) \mathfrak{U}^{\sigma \tau}{ }_{, \mu}+2 \lambda\left(\overline{\mathfrak{g}}_{\sigma \tau} \delta_{(\beta}^{\alpha} \mathfrak{U}^{\sigma \tau}{ }_{, \gamma)}-2 \overline{\mathfrak{g}}_{\sigma(\beta} \mathfrak{U}^{\alpha \sigma}{ }_{, \gamma)}\right) .
$$

We note that since $\Phi$ is a $\mathfrak{g}$-valued scalar,

$$
D_{\alpha}^{A} \Phi=\partial_{\alpha} \Phi+\left[A_{\alpha}, \Phi\right]
$$

does not involve the metric.

The stress-energy tensor can be written as

$$
\begin{gathered}
T^{\alpha \beta}=\left(g^{\alpha \mu} g^{\beta \nu}\left\langle D_{\mu}^{A} \Phi \mid D_{\nu}^{A} \Phi\right\rangle-\frac{1}{2} g^{\alpha \beta} g^{\mu \nu}\left\langle D_{\mu}^{A} \Phi \mid D_{\nu}^{A} \Phi\right\rangle\right)+ \\
\left(g^{\alpha \mu} g^{\beta \nu} g^{\sigma \tau}\left\langle F_{\mu \sigma}^{A} \mid F_{\nu \tau}^{A}\right\rangle-\frac{1}{4} g^{\mu \nu} g^{\sigma \tau} g^{\alpha \beta}\left\langle F_{\mu \sigma}^{A} \mid F_{\nu \tau}^{A}\right\rangle\right) .
\end{gathered}
$$

where $\langle\cdot \mid \cdot\rangle$ is an Ad-invariant positive definite inner-product on $\mathfrak{s u}(2)$. Using the YMH equations (2.20)-(2.21), it is straightforward to verify that any YMH solution satisfies

$$
\nabla_{\beta} T^{\alpha \beta}=0
$$

automatically irrespective of the metric. Consequently, it will be enough to solve the reduced field equations (2.18) and the YMH equations (2.20)-(2.21) to obtain a solution to the full EYMH field equations.

Let

$$
\mathcal{T}^{\alpha \beta}:=4 \pi G|\mathfrak{d}| T^{\alpha \beta}
$$

so that

$$
\begin{gathered}
\mathcal{T}^{\alpha \beta}=4 \pi G\left(\overline{\mathfrak{g}}^{\alpha \mu} \overline{\mathfrak{g}}^{\beta \nu}\left\langle D_{\mu}^{A} \Phi \mid D_{\nu}^{A} \Phi\right\rangle-\frac{1}{2} \overline{\mathfrak{g}}^{\alpha \beta} \overline{\mathfrak{g}}^{\mu \nu}\left\langle D_{\mu}^{A} \Phi \mid D_{\nu}^{A} \Phi\right\rangle\right)+ \\
\frac{4 \pi G}{\sqrt{|\mathfrak{d}|}}\left(\overline{\mathfrak{g}}^{\alpha \mu} \overline{\mathfrak{g}}^{\beta \nu} \overline{\mathfrak{g}}^{\sigma \tau}\left\langle F_{\mu \sigma}^{A} \mid F_{\nu \tau}^{A}\right\rangle-\frac{1}{4} \overline{\mathfrak{g}}^{\mu \nu} \overline{\mathfrak{g}}^{\sigma \tau} \overline{\mathfrak{g}}^{\alpha \beta}\left\langle F_{\mu \sigma}^{A} \mid F_{\nu \tau}^{A}\right\rangle\right) .
\end{gathered}
$$

\section{$3 \quad$ Weighted Sobolev Spaces}

In this section we introduce two different types of weighted Sobolev spaces and prove a number of results that will be essential to our existence proof. The following subsets of $\mathbb{R}^{n}$ will be needed: $B_{R}(x)$ the open ball of radius $R$ centered at $x \in \mathbb{R}^{n}$, $Q_{R}(x)$ the open n-cube centered at $x$ with vertices defined by the boundary of $B_{R}(x)$, and the exterior domain $E_{R}(x):=\mathbb{R}^{n} \backslash B_{R}(x)$. We will also repeatedly use the cutting function $\chi_{R} \in C_{0}^{\infty}\left(\mathbb{R}^{n}\right)$ which is defined as follows: let $\chi \in C^{\infty}[0, \infty)$ be any function such that

$$
\left.\chi\right|_{[0,1)}=1, \quad \operatorname{supp} \chi \subset[0,2), \quad \text { and } \quad 0 \leq \chi \leq 1 .
$$

Then for $R>0, \chi_{R}$ is given by

$$
\chi_{R}(x):=\chi(|x| / R) .
$$




\subsection{Radially weighted Sobolev Spaces}

Let $V$ denote a finite dimensional vector space with norm $|\cdot|$.

Definition 3.1. The radially weighted Lesbegue space $L_{\delta}^{p}\left(\mathbb{R}^{n}, V\right), 1 \leq p \leq \infty$, with weight $\delta \in \mathbb{R}$ is the set of all measurable maps from $\mathbb{R}^{n}$ to $V$ in $L_{l o c}^{p}\left(\mathbb{R}^{n}, V\right)$ such that the norm

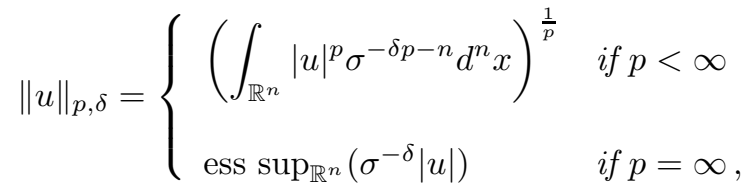

is finite. Here $\sigma(x):=\sqrt{|x|^{2}+1}$. If $V=\mathbb{R}$ then we write $L_{\delta}^{p}\left(\mathbb{R}^{n}\right)$ instead of $L_{\delta}^{p}\left(\mathbb{R}^{n}, V\right)$.

Definition 3.2. The radially weighted Sobolev space $W_{\delta}^{k, p}\left(\mathbb{R}^{n}, V\right), 1 \leq p \leq \infty$, $k \in \mathbb{N}_{0}$, with weight $\delta \in \mathbb{R}$ is the set

$$
W_{\delta}^{k, p}\left(\mathbb{R}^{n}, V\right):=\left\{u \in L_{\delta}^{p}\left(\mathbb{R}^{n}, V\right) \mid \partial^{I} u \in L_{\delta-|I|}^{p}\left(\mathbb{R}^{n}, V\right) \text { for all } I:|I| \leq k\right\}
$$

with norm

$$
\|u\|_{k, p, \delta}:=\sum_{|I| \leq k}\left\|\partial^{I} u\right\|_{p, \delta-|I|},
$$

where $I=\left(I_{1}, I_{2}, \ldots, I_{n}\right)$ is a multi-index and $\partial^{I}:=\partial_{1}^{I_{1}} \partial_{2}^{I_{2}} \cdots \partial_{n}^{I_{n}}$. If $V=\mathbb{R}$ then we will write $W_{\delta}^{k, p}\left(\mathbb{R}^{n}\right)$ instead of $W_{\delta}^{k, p}\left(\mathbb{R}^{n}, V\right)$.

We note that the set $\mathrm{C}_{0}^{\infty}\left(\mathbb{R}^{n}, V\right)$ of smooth maps from $\mathbb{R}^{n}$ to $V$ with compact support is dense in $\mathrm{W}_{\delta}^{k, p}\left(\mathbb{R}^{n}, V\right)$. As above, if $V=\mathbb{R}$ then we write $\mathrm{C}_{0}^{\infty}\left(\mathbb{R}^{n}\right)$ instead of $\mathrm{C}_{0}^{\infty}\left(\mathbb{R}^{n}, V\right)$

Two easy consequences of these definitions are that differentiation

$$
\partial_{j}: \mathrm{W}_{\delta}^{k, p}\left(\mathbb{R}^{n}, V\right) \longrightarrow \mathrm{W}_{\delta-1}^{k-1, p}\left(\mathbb{R}^{n}, V\right): u \longmapsto \partial_{j} u
$$

is a continuous map and that

$$
\mathrm{W}_{\delta_{2}}^{k, p}\left(\mathbb{R}^{n}, V\right) \subset \mathrm{W}_{\delta_{1}}^{k, p}\left(\mathbb{R}^{n}, V\right) \text { for } \delta_{2} \leq \delta_{1} .
$$

As with the Sobolev spaces, we can define weighted versions of the

$$
C_{B}^{k}\left(\mathbb{R}^{n}, V\right):=C^{k}\left(\mathbb{R}^{n}, V\right) \cap W^{k, \infty}\left(\mathbb{R}^{n}, V\right)
$$

and $C^{k, \alpha}\left(\mathbb{R}^{n}, V\right)$ spaces. For a map $u \in C^{0}\left(\mathbb{R}^{n}, V\right)$ and $\delta \in \mathbb{R}, \alpha>0$, let

$$
\|u\|_{C_{\delta}^{0}}:=\sup _{x \in \mathbb{R}^{n}}\left|\sigma(x)^{-\delta} u(x)\right|
$$


and

$$
\|u\|_{C_{\delta}^{0, \alpha}}:=\|u\|_{C_{\delta}^{0}}+\sup _{x \in \mathbb{R}^{n}}\left(\sigma^{-\delta+\alpha}(x) \sup _{4|x-y| \leq \sigma(x)} \frac{|u(x)-u(y)|}{|x-y|^{\alpha}}\right) .
$$

Using these two norms we define the norms $\|\cdot\|_{C_{\delta}^{k}}$ and $\|\cdot\|_{C_{\delta}^{k, \alpha}}$ in the usual way:

$$
\|u\|_{C_{\delta}^{k}}:=\sum_{|I| \leq k}\left\|\partial^{I} u\right\|_{C_{\delta-|I|}^{0}}
$$

and

$$
\|u\|_{C_{\delta}^{k, \alpha}}:=\sum_{|I| \leq k}\left\|\partial^{I} u\right\|_{C_{\delta-|I|}^{0, \alpha}}
$$

So then

$$
C_{\delta}^{k}\left(\mathbb{R}^{n}, V\right):=\left\{u \in C^{k}\left(\mathbb{R}^{n}, V\right) \mid\|u\|_{C_{\delta}^{k}}<\infty\right\}
$$

and

$$
C_{\delta}^{k, \alpha}\left(\mathbb{R}^{n}, V\right):=\left\{u \in C^{k}\left(\mathbb{R}^{n}, V\right) \mid\|u\|_{C_{\delta}^{k, \alpha}}<\infty\right\} .
$$

Our main references for the radially weighted Sobolev spaces will be [1] and [5]. Contained in these articles are a number useful theorems including weighted versions of the Sobolev embedding theorems, the Rellich-Kondrachov theorem, and interior estimates for elliptic operators. Also contained in these papers in an analysis of the Laplace operator and its mapping properties between the radially weighted spaces. We will frequently require results from these papers and will refer the reader to the appropriate theorems. A result we would like to mention is the following improvement of lemma 2.5 of [5].

Lemma 3.3. If there exists a multiplication $V_{1} \times V_{2} \rightarrow V_{3}(u, v) \mapsto u \cdot v$ then for $1 \leq p<\infty$ the corresponding multiplication

$$
W_{\delta_{1}}^{k_{1}, p}\left(\mathbb{R}^{n}, V_{1}\right) \times W_{\delta_{2}}^{k_{2}, p}\left(\mathbb{R}^{n}, V_{2}\right) \rightarrow W_{\delta_{3}}^{k_{3}, p}\left(\mathbb{R}^{n}, V_{3}\right):(u, v) \mapsto u \cdot v
$$

is bilinear and continuous if $k_{1}, k_{2} \geq k_{3}, k_{3}<k_{1}+k_{2}-n / p$, and $\delta_{1}+\delta_{2} \leq \delta_{3}$.

Proof. This can be proved using the weighted Sobolev and Hölder inequalities from theorem 1.2 of [1] in exactly the same fashion as for the regular unweighted Sobolev spaces. Note that theorem 1.2 of [1] is missing the weighted version of the Sobolev inequality for $k p=n$. The same arguments in theorem 1.2 can be used to establish this case which reads: if $u \in \mathrm{W}_{\delta}^{k, p}$ and $n=k p$, then $\|u\|_{q, \delta} \leq C\|u\|_{p, k, \delta}$ for $p \leq q<\infty$.

We also will need the following variation of proposition 1.6 of [1].

Proposition 3.4. Suppose $1<p<\infty$ and $\delta \in \mathbb{R}$ and $f(x)$ is a continuous function that satisfies $f(x)=O\left(|x|^{-2}\right)$ as $|x| \rightarrow \infty$. Then there exists a constant $C$ such that if $u \in L_{\delta}^{0, p}$ and $\Delta u+f u \in L_{\delta-2}^{0, p}$ then $u \in W_{\delta}^{2, p}$ and

$$
\|u\|_{2, p, \delta} \leq C\left(\|\Delta u+f u\|_{0, p, \delta-2}+\|u\|_{0, p, \delta}\right) .
$$


Proof. This proof follows from the local elliptic estimates and scaling in exactly the same fashion as the proof of proposition 1.6 in [1].

\subsection{Exponentially weighted Sobolev Spaces}

Definition 3.5. The exponentially weighted Lesbegue space $\mathcal{L}_{\mu}^{p}\left(\mathbb{R}^{n}, V\right), 1 \leq p \leq$ $\infty$, with weight $\mu \in \mathbb{R}$ is the set of all measurable maps from $\mathbb{R}^{n}$ to $V$ in $\mathcal{L}_{\text {loc }}^{p}\left(\mathbb{R}^{n}, V\right)$ such that the norm

$$
\|u\|_{p, \mu}= \begin{cases}\left(\int_{\mathbb{R}^{n}}|u(x)|^{p} e^{-\mu p|x|} d^{n} x\right)^{\frac{1}{p}} & \text { if } p<\infty \\ \operatorname{ess}_{\sup _{\mathbb{R}^{n}}\left(e^{-\mu|x|}|u(x)|\right)} & \text { if } p=\infty,\end{cases}
$$

is finite. If $V=\mathbb{R}$ then we write $\mathcal{L}_{\mu}^{p}\left(\mathbb{R}^{n}\right)$ instead of $\mathcal{L}_{\mu}^{p}\left(\mathbb{R}^{n}, V\right)$.

Definition 3.6. The exponentially weighted Sobolev space $\mathcal{W}_{\mu}^{k, p}\left(\mathbb{R}^{n}, V\right), 1 \leq p \leq$ $\infty, k \in \mathbb{N}_{0}$, with weight $\mu \in \mathbb{R}$ is the set

$$
\mathcal{W}_{\mu}^{k, p}\left(\mathbb{R}^{n}, V\right):=\left\{u \in \mathcal{L}_{\mu}^{p}\left(\mathbb{R}^{n}, V\right) \mid \partial^{I} u \in \mathcal{L}_{\mu}^{p}\left(\mathbb{R}^{n}, V\right) \text { for all } I:|I| \leq k\right\}
$$

with norm

$$
\|u\|_{k, p, \mu}:=\sum_{|I| \leq k}\left\|\partial^{I} u\right\|_{p, \mu} .
$$

If $V=\mathbb{R}$ then we will write $\mathcal{W}_{\delta}^{k, p}\left(\mathbb{R}^{n}\right)$ instead of $\mathcal{W}_{\mu}^{k, p}\left(\mathbb{R}^{n}, V\right)$.

We note that the $\mathrm{C}_{0}^{\infty}\left(\mathbb{R}^{n}, V\right)$ is dense in $\mathcal{W}_{\mu}^{k, p}\left(\mathbb{R}^{n}, V\right)$. A straightforward consequence of the above definitions is that differentiation

$$
\partial_{j}: \mathcal{W}_{\mu}^{k, p}\left(\mathbb{R}^{n}, V\right) \longrightarrow \mathcal{W}_{\mu}^{k-1, p}\left(\mathbb{R}^{n}, V\right): u \longmapsto \partial_{j} u
$$

is a continuous map. Also note that $\mathcal{W}_{0}^{k, p}\left(\mathbb{R}^{n}, V\right)=\mathrm{W}^{k, p}\left(\mathbb{R}^{n}, V\right)$ while it follows from [1] theorem 1.2 (i) that $\mathrm{W}_{\delta}^{k, p}\left(\mathbb{R}^{n}, V\right) \subset \mathrm{W}^{k, p}\left(\mathbb{R}^{n}, V\right)$ for $\delta \leq-n / p$. Consequently we have the inclusion

$$
\mathrm{W}_{\delta}^{k, p}\left(\mathbb{R}^{n}, V\right) \subset \mathcal{W}_{0}^{k, p}\left(\mathbb{R}^{n}, V\right) \text { for } \delta \leq-n / p .
$$

It also follows directly from Hölders inequality and the definitions of the radially and exponentially weighted spaces that

$$
\mathcal{W}_{\mu}^{k, p}\left(\mathbb{R}^{n}, V\right) \subset \mathrm{W}_{\delta}^{k, p}\left(\mathbb{R}^{n}, V\right) \quad \text { for all } \delta \in \mathbb{R} \text { provided } \mu<0 .
$$

As with the radially weighted case, we can also define the corresponding exponential weighted $C_{B}^{k}\left(\mathbb{R}^{n}, V\right)$ and $C^{k, \alpha}\left(\mathbb{R}^{n}, V\right)$ spaces. For a map $u \in C^{0}\left(\mathbb{R}^{3}, V\right)$ and $\delta \in \mathbb{R}, \alpha>0$, let

$$
\|u\|_{\mathcal{C}_{\mu}^{0}}:=\sup _{x \in \mathbb{R}^{n}}\left|e^{-\mu|x|} u(x)\right|
$$


and

$$
\|u\|_{\mathcal{C}_{\mu}^{0, \alpha}}:=\|u\|_{\mathcal{C}_{\mu}^{0}}+\sup _{x \in \mathbb{R}^{n}}\left(e^{-\mu|x|} \sup _{|x-y| \leq 1} \frac{|u(x)-u(y)|}{|x-y|^{\alpha}}\right) .
$$

Using these two norms we define the norms $\|\cdot\|_{\mathcal{C}_{\mu}^{k}}$ and $\|\cdot\|_{\mathcal{C}_{\mu}^{k, \alpha}}$ by

$$
\|u\|_{\mathcal{C}_{\mu}^{k}}:=\sum_{|I| \leq k}\left\|\partial^{I} u\right\|_{\mathcal{C}_{\mu}^{0}}
$$

and

$$
\|u\|_{\mathcal{C}_{\mu}^{k, \alpha}}:=\sum_{|I| \leq k}\left\|\partial^{I} u\right\|_{\mathcal{C}_{\mu}^{0, \alpha}}
$$

So then

$$
\mathcal{C}_{\mu}^{k}\left(\mathbb{R}^{n}, V\right):=\left\{u \in C^{k}(\mathbb{R}, V) \mid\|u\|_{\mathcal{C}_{\mu}^{k}}<\infty\right\}
$$

and

$$
\mathcal{C}_{\mu}^{k, \alpha}\left(\mathbb{R}^{n}, V\right):=\left\{u \in C^{k}(\mathbb{R}, V) \mid\|u\|_{\mathcal{C}_{\mu}^{k, \alpha}}<\infty\right\} .
$$

To prove weighted versions of the Sobolev inequalities from local inequalities, a covering argument is needed. Let $\left\{x_{a}\right\}_{a \in \mathbb{Z}^{n}}$ be a sequence of points such that

$$
\mathbb{R}^{n}=\bigcup_{a \in \mathbb{Z}^{n}} \overline{Q_{R}\left(x_{a}\right)}
$$

and $Q_{R}\left(x_{a}\right) \cap Q_{R}\left(x_{a^{\prime}}\right)=\emptyset$ for $a \neq a^{\prime}$. Then there exists a number $N$ independent of $a$ such that the set

$$
\left\{a^{\prime} \in \mathbb{Z}^{n} \mid B_{2 R}\left(x_{a}\right) \cap Q_{R}\left(x_{a^{\prime}}\right) \neq \emptyset\right\}
$$

has at most $N$ elements. The key property we need is that for any $\sigma \in \mathbb{R}$ there exists a constant $C=C(\sigma, R)$ independent of $x \in \mathbb{R}^{n}$ such that

$$
C^{-1} e^{\sigma|x|} \leq e^{\sigma|y|} \leq C e^{\sigma|x|} \quad \forall y \in B_{R}(x) .
$$

From this inequality it follows that there exists a constant $C$ independent of $x$ such that

$$
C^{-1} e^{-\mu|x|}\left\|u_{x}\right\|_{k, p ; B_{R}(0)} \leq\|u\|_{k, p, \mu ; B_{R}(x)} \leq C e^{-\mu|x|}\left\|u_{x}\right\|_{k, p ; B_{R}(0)}
$$

where

$$
u_{x}(y):=u(x+y) .
$$

Note that the constant only depends on $\mu, p, k$ and $R$. Equations (3.8)-(3.12) will allow us to turn local estimates into global ones. The next theorem generalizes the Hölder and Sobolev inequalities to the exponentially weighted spaces and the proof closely follows that of theorem 1.2 of [1]. 
Theorem 3.7. (i) If $1 \leq p \leq q \leq \infty, \mu_{2}<\mu_{1}$ and $u \in \mathcal{L}_{\mu_{2}}^{q}$, then

$$
\|u\|_{p, \mu_{1}} \leq C\|u\|_{q, \mu_{2}}
$$

and hence $\mathcal{L}_{\mu_{2}}^{q} \subset \mathcal{L}_{\mu 1}^{p}$.

(ii) If $1 \leq p, q, r \leq \infty, \frac{1}{r}=\frac{1}{q}+\frac{1}{p}, u \in \mathcal{L}_{\mu_{1}}^{q}, v \in \mathcal{L}_{\mu_{2}}^{p}$, and $\mu_{3}=\mu_{1}+\mu_{2}$ then

$$
\|u v\|_{r, \mu_{3}} \leq\|u\|_{q, \mu 1}\|v\|_{p, \mu_{2}} .
$$

(iii) For any $\epsilon>0$, there is a $C(\epsilon)$ such that for all $u \in \mathcal{W}_{\mu}^{2, p}, 1 \leq p \leq \infty$,

$$
\|u\|_{1, p, \mu} \leq \epsilon\|u\|_{2, p, \mu}+C(\epsilon)\|u\|_{0, p, \mu} .
$$

(iv) If $u \in \mathcal{W}_{\mu}^{k, p}$ and $n-k p>0$ then

$$
\|u\|_{q, \mu} \leq C\|u\|_{k, p, \mu}
$$

for $p \leq q \leq n p /(n-k p)$.

(v) If $u \in \mathcal{W}_{\mu}^{k, p}$ and $n-k p=0$ then

$$
\|u\|_{q, \mu} \leq C\|u\|_{k, p, \mu}
$$

for $p \leq q<\infty$.

(vi) If $u \in \mathcal{W}_{\mu}^{k, p}$ and $n-k p<0$ then $u \in \mathcal{C}_{\mu}^{0}$ and

$$
\|u\|_{\mathcal{C}_{\mu}^{0}} \leq C\|u\|_{k, p, \mu} .
$$

$$
\text { Moreover }|u(x)|=o\left(e^{\mu|x|}\right) \text { as }|x| \rightarrow \infty \text {. }
$$

(vii) If $u \in \mathcal{W}_{\mu}^{k, p}, 0<\alpha \leq k-n / p \leq 1$, then $u \in \mathcal{C}_{\mu}^{0, \alpha}$ and

$$
\|u\|_{\mathcal{C}_{\mu}^{0, \alpha}} \leq C\|u\|_{k, p, \mu} .
$$

Proof. Parts (i) and (ii) follow from the definition and Hölder's inequality. The proofs of (iii)-(vii) follow from the interpolation and Sobolev inequalities on $B_{1}(0)$ together with equations (3.8)-(3.12). We will only prove (iv) and leave the remainder to the reader. So assume that $n-p k>0, p \leq q \leq n p /(n-k p)$, and $u \in \mathcal{W}_{\mu}^{k, p}$. Then clearly $u_{x} \in \mathrm{W}^{k, p}\left(B_{2}(0)\right)$ and hence applying the standard Sobolev inequality yields

$$
\left\|u_{x}\right\|_{q ; B_{2}(0)} \leq C\left\|u_{x}\right\|_{p, k ; B_{2}(0)}=C\|u\|_{p, k ; B_{2}(x)} .
$$

The constant $C$ above only depends on $p, k$, and the ball $B_{2}(0)$. Using (3.11) we get

$$
\|u\|_{q, \mu ; B_{2}(x)} \leq C\|u\|_{p, k, \mu ; B_{2}(x)}
$$


for a constant $C$ independent of $u$ and $x$. So

$$
\begin{aligned}
\|u\|_{q, \mu} & \leq\left(\sum_{a \in \mathbb{Z}^{n}}\|u\|_{q, \mu ; B_{2}\left(x_{a}\right)}^{q}\right)^{1 / q} \\
& \leq C\left(\sum_{a \in \mathbb{Z}^{n}}\|u\|_{p, k, \mu ; B_{2}\left(x_{a}\right)}^{q}\right)^{1 / q} \quad \text { by (3.13) } \\
& \leq C\left(\sum_{a \in \mathbb{Z}^{n}}\|u\|_{p, k, \mu ; B_{2}\left(x_{a}\right)}^{p}\right)^{1 / p}
\end{aligned}
$$

where in deriving the last inequality we have used $\left(\sum_{j} b_{j}^{s}\right)^{1 / s} \leq\left(\sum_{j} b_{j}^{t}\right)^{1 / t}$ for $b_{j} \geq 0$ and $t \leq s$. Using the finite intersection property (3.9), there exists a constant $K$ independent of $u$ such that

$$
\sum_{a \in \mathbb{Z}^{n}}\|u\|_{p, k, \mu ; B_{2}\left(x_{a}\right)}^{p} \leq K\|u\|_{p, k, \mu}^{p}
$$

To see this it is enough to show it for the norm $\|\cdot\|_{p, \mu}$. From the finite interesction property we know that there exists a set of points $\left\{x_{a_{1}}=x_{a}, x_{a_{2}}, \ldots, x_{a_{N}}\right\}$ such that

$$
B_{2}\left(x_{a}\right) \subset E \cup \bigcup_{j=1}^{N} Q_{1}\left(x_{a_{j}}\right)
$$

where $E$ is a set of measure zero. So

$$
\|u\|_{p, \mu ; B_{2}\left(x_{a}\right)}^{p}=\int_{B_{2}\left(x_{a}\right)}|u(x)|^{p} e^{-\mu p|x|} d^{n} x \leq \sum_{j=1}^{N} \int_{Q_{1}\left(x_{a_{j}}\right)}|u(x)|^{p} e^{-\mu p|x|} d^{n} x
$$

and hence

$$
\begin{aligned}
& \sum_{a \in \mathbb{Z}^{n}} \| \\
& \quad \leq N \|_{p, \mu ; B_{2}\left(x_{a}\right)}^{p} \leq \sum_{a \in \mathbb{Z}^{n}} \sum_{j=1}^{N} \int_{Q_{1}\left(x_{a_{j}}\right)}|u(x)|^{p} e^{-\mu p|x|} d^{n} x \\
& \quad \leq N \int_{a \in \mathbb{Z}^{n}} \int_{Q_{1}\left(x_{a}\right)}|u(x)|^{p} e^{-\mu p|x|} d^{n} x=N \int_{\mathbb{R}^{n}}|u(x)|^{p} e^{-\mu p|x|} d^{n} x=N\|u\|_{p, \mu} .
\end{aligned}
$$

Note in getting the second to last equality we use the fact that the set

$$
\mathbb{R}^{n} \backslash\left(\bigcup_{a \in \mathbb{Z}^{n}} Q_{1}\left(x_{a}\right)\right)
$$

has measure zero. Equation (3.15) now follows immediately. Combining (3.14) and (3.15) proves (iii). 
The first of the following two lemmas is the exponentially weighted version of the Rellich-Kondrachov theorem and both lemmas can be proved by adapting the proof of lemma 2.1 in [5]. We only prove the second and leave the first to the reader.

Lemma 3.8. For $k_{1}>k_{2}, \mu_{1}<\mu_{2}$ and $1 \leq p<\infty$ the inclusion $\mathcal{W}_{\mu_{1}}^{k_{1}} \subset \mathcal{W}_{\mu_{2}}^{k_{2}}$ is compact.

Lemma 3.9. Suppose $v \in W^{\infty, k_{1}}\left(\mathbb{R}^{n}\right)$ and the function

$$
\xi(R):=\max _{0 \leq|I| \leq k_{1}} \sup _{x \in E_{R}(0)}\left|\partial^{I} v(x)\right|
$$

satisfies $\lim _{R \rightarrow \infty} \xi(R)=0$. Then for $k_{1}>k_{2}$ and $1 \leq p<\infty$ the map

$$
\mathcal{W}_{\mu}^{k_{1}} \longrightarrow \mathcal{W}_{\mu}^{k_{2}, p}: u \longmapsto v u
$$

is compact.

Proof. Let $\left\{u_{n}\right\} \in \mathcal{W}_{\mu_{1}}^{k_{1}, p}$ be a sequence such that $\left\|u_{n}\right\|_{k_{1}, p, \mu} \leq 1$. Then there exists a subsequence still denoted $\left\{u_{n}\right\}$ such that $u_{n} \rightarrow u$ weakly in $\mathcal{W}_{\mu}^{k_{1}, p}$ for some $u \in \mathcal{W}_{\mu}^{k_{1}, p}$ with $\|u\|_{k_{1}, p, \mu} \leq 1$. From theorem 3.7 (ii) we have that $\|v u\|_{k_{1}, p, \mu} \leq$ $C\|u\|_{k_{1}, p, \mu}$ for some $C$ that depends only on $\xi(R)$. Therefore the map

$$
L_{v}: \mathcal{W}_{\mu}^{k_{1}, p} \longrightarrow \mathcal{W}_{\mu}^{k_{1}, p} ; u \longmapsto v u
$$

is continuous and hence weakly continuous. So $v u_{n} \rightarrow v u$ weakly in $\mathcal{W}_{\mu}^{k_{1}, p}$. By (3.10) there exist a constant $C_{R}$ depending only on $\mu, p$ and $\left\|\chi_{R}\right\|_{C^{k_{1}\left(B_{R}(0)\right)}}$ such that

But then

$$
\left\|\chi_{R} v u_{n}\right\|_{k_{1}, p ; B_{2}(0)} \leq C_{R}\left\|v u_{n}\right\|_{k_{1}, p, \mu} .
$$

$$
\left\|\chi_{R} v u_{n}\right\|_{k_{1}, p ; B_{2 R}(0)} \leq C_{R}\left\|L_{v}\right\|_{\mathrm{op}}
$$

by (3.16), (3.17), and $\left\|u_{n}\right\|_{k_{1}, p, \mu} \leq 1$, where $\left\|L_{v}\right\|_{\text {op }}$ denotes the operator norm of $L_{v}$ The compactness of the embedding $\mathrm{W}^{k_{1}, p}\left(B_{2 R}(0)\right) \rightarrow \mathrm{W}^{k_{2}, p}\left(B_{2 R}(0)\right)\left(k_{1}>k_{2}\right)$ shows that there exist a subsequence $\left\{\chi_{R} v u_{n_{i}}\right\}$ such that

$$
\chi_{R} v u_{n_{i}} \rightarrow f_{R} \quad \text { strongly in } \mathrm{W}^{k_{2}, p}\left(B_{2 R}(0)\right)
$$

for some $f_{R}$ in $\mathrm{W}^{k_{2}, p}\left(B_{2 R}(0)\right)$. Since $\chi_{R}=1$ on $B_{R}(0)$ we must have that

$$
f_{R}=v u \quad \text { on } B_{R}(0) .
$$

Setting $c_{R}=\sup _{r \in[0, R]} e^{-\mu p r}$, we get

$$
\begin{aligned}
\left\|v u-v u_{n_{i}}\right\|_{k_{2}, p, \mu}^{p} & \leq c_{R}\left\|v u-v u_{n_{i}}\right\|_{k_{2}, p ; B_{R}(0)}^{p}+K \xi(R)^{p}\left\|u-u_{n_{i}}\right\|_{k_{2}, p, \mu ; E_{R}}^{p} \\
& \leq c_{R}\left\|v u-v u_{n_{i}}\right\|_{k_{2}, p ; B_{R}(0)}^{p}+K \xi(R)^{p}\left\|u-u_{n_{i}}\right\|_{k_{1}, p, \mu}^{p} \\
& \leq c_{R}\left\|v u-v u_{n_{i}}\right\|_{k_{2}, p ; B_{R}(0)}^{p}+2^{p} K \xi(R)^{p}
\end{aligned}
$$


where $\mathrm{K}$ is a constant independent of $\mathrm{R}$ and in getting the last inequality we used $\left\|u_{n}\right\|_{k_{1}, p, \mu},\|u\|_{k_{1}, p, \mu} \leq 1$. For fixed $\epsilon>0$ we can choose $R$ large enough so that

$$
2^{p} K \xi(R)^{p} \leq \frac{\epsilon^{p}}{2} .
$$

With $R$ fixed, we get by (3.18) and (3.19) that there exists an $M>0$ such that

$$
c_{R}\left\|v u-v u_{n_{i}}\right\|_{k_{2}, p ; B_{R}(0)}^{p} \leq \frac{\epsilon^{p}}{2} \quad \text { for } i \geq M .
$$

Therefore $\left\|v u-v u_{n_{i}}\right\|_{k_{2}, p, \mu} \leq \epsilon$ for $i \geq M$ and hence $v u_{n_{i}}$ converges to $v u$ in $\mathcal{W}_{\mu}^{k_{2}, p}$. This proves that the map $L_{v}(u)=u v$ is compact.

The exponentially weighted Sobolev and Hölder inequalities can also be used to prove a multiplication lemma as in the radially weighted case (see lemma 3.3).

Lemma 3.10. If there exists a multiplication $V_{1} \times V_{2} \rightarrow V_{3}(u, v) \mapsto u \cdot v$ then for $1 \leq p<\infty$ the corresponding multiplication

$$
\mathcal{W}_{\mu_{1}}^{k_{1}, p}\left(\mathbb{R}^{n}, V_{1}\right) \times \mathcal{W}_{\mu_{2}}^{k_{2}, p}\left(\mathbb{R}^{n}, V_{2}\right) \rightarrow \mathcal{W}_{\mu_{3}}^{k_{3}, p}\left(\mathbb{R}^{n}, V_{3}\right):(u, v) \mapsto u \cdot v
$$

is bilinear and continuous if $k_{1}, k_{2} \geq k_{3}, k_{3}<k_{1}+k_{2}-n / p$, and $\mu_{1}+\mu_{2} \leq \mu_{3}$.

As with the local Sobolev inequaltites, local estimates for elliptic operators can be extended to global ones on the exponentially weighted spaces.

Proposition 3.11. Let $1<p<\infty$, and $P$ be the elliptic operator defined by

$$
P u=a^{i j} \partial_{i j}^{2} u+b^{i}(x) \partial_{i} u+c(x) u
$$

where $b^{i}, c \in L^{\infty}\left(\mathbb{R}^{n}\right)$ and there exists constants $\lambda>0,0<\alpha \leq 1$ such that $a^{i j} \in$ $C_{0}^{0, \alpha}\left(\mathbb{R}^{n}\right)$ and $\lambda|\xi|^{2} \leq a^{i j}(x) \xi_{i} \xi_{j} \leq \lambda^{-1}|\xi|^{2}$ for all for all $x, \xi \in \mathbb{R}^{n}$. Then $P$ defines a continuous map from $\mathcal{W}_{\mu}^{2, p} \rightarrow \mathcal{W}_{\mu}^{0, p}$. Moreover, if $u \in \mathcal{W}_{\mu}^{0, p}$ and $P u \in \mathcal{W}_{\mu}^{0, p}$ then $u \in \mathcal{W}_{\mu}^{2, p}$ and there exists a constant $C=C\left(n, p, \lambda,\left\|a^{i j}\right\|_{C_{0}^{0, \alpha}},\left\|b^{i}\right\|_{\infty},\|c\|_{\infty}\right)$ such that

$$
\|u\|_{2, p, \mu} \leq C\left(\|P u\|_{0, p, \mu}+\|u\|_{0, p, \mu}\right) .
$$

Proof. If $u \in \mathcal{W}_{\mu}^{0, p}$ and $P u \in \mathcal{W}_{\mu}^{0, p}$, then elliptic regularity shows that $u \in \mathcal{W}_{\text {loc }}^{2, p}$. The proof then follows from the local elliptic estimates (see [8], theorem 9.11) and the covering argument.

In the analysis of elliptic operators on the radially weighted spaces the Laplacian $\Delta$ played a fundamental role. The corresponding fundamental elliptic operator on the exponentially weighted spaces is

$$
-\Delta+\kappa^{2} \quad \text { where } \kappa>0 \text { is a constant. }
$$


With our applications in mind, we will restrict ourselves to $n=3$ for the remainder of this section. The operator (3.20) has a Green's function $G_{\kappa}(x, y)$ which for $n=3$ is

$$
G_{\kappa}(x, y)=G_{\kappa}(x-y)=\frac{1}{4 \pi} \frac{e^{-\kappa|x-y|}}{|x-y|}
$$

and is known as the Yukawa potential. It satisfies the distributional identity

$$
\left(-\Delta_{x}+\kappa^{2}\right) G_{\kappa}(x, y)=\delta(x-y) \quad \text { in } \mathcal{D}^{\prime}\left(\mathbb{R}^{3}\right) .
$$

The invertibility of the operator (3.20) can be established from an estimate for the Green's function combined with the weighted elliptic estimates in a similar fashion as for the Laplacian [1].

Theorem 3.12. If $\kappa-|\mu|>0,1<p<\infty$, and $s$ is a non-negative integer then the operator

$$
-\Delta+\kappa^{2}: \mathcal{W}_{\mu}^{2+s, p} \longrightarrow \mathcal{W}_{\mu}^{s, p}
$$

is an isomorphism with the inverse given by

$$
\left(-\Delta+\kappa^{2}\right)^{-1} u(x)=\frac{1}{4 \pi} \int_{\mathbb{R}^{3}} \frac{e^{-\kappa|x-y|}}{|x-y|} u(y) d y .
$$

Proof. It suffices to prove the theorem for $s=0$. Let $\hat{G}_{\kappa}$ be the operator defined by

$$
\hat{G}_{\kappa}(u)(x):=\int_{\mathbb{R}^{3}} G_{\kappa}(x-y) u(y) d y .
$$

Lemma 3.13. If $p \geq 1, \kappa-|\mu|>0$ and $u \in \mathcal{L}_{\mu}^{p}$, then

$$
\left\|\hat{G}_{\kappa}(u)\right\|_{p, \mu} \leq C\|u\|_{p, \mu}
$$

for a constant $C$ independent of $u$.

Proof. For all $\mu \in \mathbb{R}$ and $x, y \in \mathbb{R}^{3}$ it holds that $\mu|y|-\mu|x| \leq|\mu||x-y|$ and hence

$$
e^{\mu|y|-\mu|x|} \leq e^{|\mu||x-y|} .
$$

Using this and the definition of the Green's function (3.21), we see that for two non-negative functions $u, v$

$$
0 \leq u(x) e^{-\mu|x|} G_{\kappa}(x-y) e^{\mu|y|} v(y) \leq u(x) G_{\kappa-|\mu|}(x-y) v(y) .
$$

Integrating gives

$$
\begin{aligned}
& \int_{\mathbb{R}^{3}} \int_{\mathbb{R}^{3}} u(x) e^{-\mu|x|} G_{\kappa}(x-y) e^{\mu|y|} v(y) d x d y \\
& \leq \int_{\mathbb{R}^{3}} \int_{\mathbb{R}^{3}} u(x) G_{\kappa-|\mu|}(x-y) v(y) d x d y .
\end{aligned}
$$


Noting that $G_{\kappa-|\mu|} \in L^{1}\left(\mathbb{R}^{3}\right)$ for $\kappa-|\mu|>0$, Young's inequality (see [12], theorem 4.2) applied to (3.26) yields

$$
\int_{\mathbb{R}^{3}} \int_{\mathbb{R}^{3}} u(x) e^{-\mu|x|} G_{\kappa}(x-y) e^{\mu|y|} v(y) d x d y \leq C\|u\|_{p^{\prime}}\left\|G_{\kappa-|\mu|}\right\|_{1}\|v\|_{p}
$$

where $\frac{1}{p}+\frac{1}{p^{\prime}}=1, p \geq 1$. Setting

$$
u(x)=\left(\int_{\mathbb{R}^{3}} e^{-\mu|x|} G_{\kappa}(x-y) e^{\mu|y|} v(y) d y\right)^{p / p^{\prime}}
$$

in (3.27) yields

$$
\left(\int_{\mathbb{R}^{3}}\left|\int_{\mathbb{R}^{3}} e^{-\mu|x|} G_{\kappa}(x-y) e^{\mu|y|} v(y) d y\right|^{p} d x\right)^{1 / p} \leq C\left\|G_{\kappa-|\mu|}\right\|_{1}\|v\|_{p} .
$$

Finally, setting $v(y)=e^{-\mu|y|} w(y)$ in (3.28) shows that

$$
\left\|\hat{G}_{\kappa}(w)\right\|_{p, \mu} \leq C\left\|G_{\kappa-|\mu|}\right\|_{1}\|w\|_{p, \mu} .
$$

So far our above choices amount to assuming that $w \geq 0$. However, it is clear that the above inequality extends to all $w \in \mathcal{L}_{\mu}^{p}$.

The distributional identity (3.22) shows that $\hat{G}_{\kappa}\left(\Delta u-\kappa^{2} u\right)=-u$ for all $u \in$ $\mathrm{C}_{0}^{\infty}\left(\mathbb{R}^{3}\right)$, and hence

$$
\|u\|_{0, p, \mu} \leq C\left\|\Delta u-\kappa^{2} u\right\|_{0, p, \mu} \quad \text { for all } u \in \mathcal{W}_{\mu}^{0, p}
$$

by lemma 3.13 and the density of $\mathrm{C}_{0}^{\infty}\left(\mathbb{R}^{3}\right)$ in $\mathcal{W}_{\mu}^{0, p}$. Applying proposition (3.11) to the above inequality then yields

$$
\|u\|_{2, k, p} \leq C\left\|\Delta u-\kappa^{2} u\right\|_{0, p, \mu} \quad \text { for all } u \in \mathcal{W}_{\mu}^{0, p} .
$$

Since $-\Delta+\kappa^{2}: \mathcal{W}_{\mu}^{2, p} \rightarrow \mathcal{W}_{\mu}^{0, p}$ is bounded, it follows easily from (3.29) that $-\Delta+\kappa^{2}$ has closed range and a trivial kernel. The distributional identity (3.22) implies that $\left(-\Delta+\kappa^{2}\right) \hat{G}_{\kappa}(u)=u$ for all $u \in \mathrm{C}_{0}^{\infty}\left(\mathbb{R}^{3}\right)$. But by lemma $2.1 \hat{G}_{\kappa}(u) \in \mathcal{W}_{\mu}^{0, p}$ and hence $\hat{G}_{\kappa}(u) \in \mathcal{W}_{\mu}^{2, p}$ by proposition 3.11 Therefore $-\Delta+\kappa^{2}$ is surjective.

\section{Static spherically symmetric fields}

We assume that all the fields are static and that $\partial_{0}$ is a timelike hypersurface orthogonal killing vector field for the metric. Therefore

$$
\partial_{0} \mathfrak{U}^{\alpha \beta}=0, \partial_{0} A_{\alpha}=0, \partial_{0} \Phi=0 \quad \text { and } \quad \mathfrak{U}^{j 0}=\mathfrak{U}^{0 j}=0 .
$$


Since $\mathfrak{U}^{\alpha \beta}$ is symmetric, i.e. $\mathfrak{U}^{\alpha \beta}=\mathfrak{U}^{\beta \alpha}$, we define the following subspace of the 4 by 4 matrices

$$
\mathbb{S}:=\left\{X=\left(X^{\alpha \beta}\right) \in \mathbb{M}_{4 \times 4} \mid X^{\alpha \beta}=X^{\beta \alpha} \text { and } X^{0 j}=0\right\} .
$$

Then letting $\mathfrak{U}=\left(\mathfrak{U}^{\alpha \beta}\right), \mathfrak{U}$ takes values in $\mathbb{S}$.

In addition to being static, we will also assume that our fields are spherically symmetric. To define what we mean by spherical symmetry we first need to specify an action of $S O(3)$ on spacetime $\mathbb{R}^{4}$. We want $S O(3)$ to act on the hypersurfaces orthogonal to the timelike Killing vector field $\partial_{0}$. So using the matrix representation of $S O(3)$ given by

$$
S O(3)=\left\{a \in \mathbb{M}_{3 \times 3} \mid a^{t}=a^{-1} \text { and } \operatorname{det}(a)=1\right\}
$$

we define a $S O(3)$ action on spacetime by

$$
\rho: S O(3) \times \mathbb{R}^{4} \rightarrow \mathbb{R}^{4}:\left(a,\left(x^{0}, x\right)\right) \rightarrow \Phi_{a}\left(x^{0}, x\right):=\left(x^{0}, a x\right)
$$

where we are treating $x$ as a column vector and $a x$ denotes matrix multiplication. We then get the induced action on functions via pullbacks. Lifting the $S O(3)$ action on spacetime to the tensor bundle, we get the following action on the static metric densities

$$
\left.\rho_{a}(\mathfrak{U})(x):=\tilde{a} \mathfrak{U}\left(a^{\mathrm{t}} x\right)\right) \tilde{a}^{\mathrm{t}}
$$

where

$$
\tilde{a}:=\left(\begin{array}{ll}
1 & 0 \\
0 & a
\end{array}\right) .
$$

This allows us to define the set of static smooth $S O(3)$-invariant metric densities by

$$
\mathcal{U}_{0}^{\infty}:=\left\{\mathfrak{U} \in \mathrm{C}_{0}^{\infty}\left(\mathbb{R}^{3}, \mathbb{S}\right) \mid \mathfrak{U}=\rho_{a} \mathfrak{U} \text { for all } a \in S O(3)\right\} .
$$

Completing in the $\mathrm{W}_{\delta}^{k, p}$ norm yields

$$
\mathcal{U}_{\delta}^{k, p}:=\overline{\mathcal{U}_{0}^{\infty}} \subset \mathrm{W}_{\delta}^{k, p}\left(\mathbb{R}^{3}, \mathbb{S}\right) .
$$

Proposition 4.1. For $-1<\delta<0,1<p<\infty$ and $k \in \mathbb{N}_{0}$ the Laplacian $\Delta: \mathcal{U}_{\delta}^{k+2, p} \rightarrow \mathcal{U}_{\delta-2}^{k, p}$ is an isomorphism.

Proof. From proposition 2.2 of [1] we have that $\Delta: \mathrm{W}_{\delta}^{k, p}\left(\mathbb{R}^{3}, \mathbb{S}\right) \rightarrow \mathrm{W}_{\delta-2}^{k-2, p}\left(\mathbb{R}^{3}, \mathbb{S}\right)$ is an isomorphism for $1<p<\infty,-1<\delta<0$. A straightforward calculation shows that $\Delta\left(\mathcal{U}_{0}^{\infty}\right) \subset \mathcal{U}_{0}^{\infty}$. Similarly, using the formula

$$
\left(\Delta^{-1} \mathfrak{U}^{\alpha \beta}\right)(x)=\frac{-1}{4 \pi} \int_{\mathbb{R}^{3}} \frac{\mathfrak{U}^{\alpha \beta}(y)}{|x-y|} d^{3} y
$$

it is not difficult to verify that if $\mathfrak{U} \in \mathcal{U}_{0}^{\infty}$ then $\rho_{a}\left(\Delta^{-1} \mathfrak{U}\right)=\Delta^{-1} \mathfrak{U}$ for all $a \in S O(3)$. But $\mathcal{U}_{0}^{\infty}$ is dense in $\mathcal{U}_{\delta}^{k, p}$ and hence the proof follows. 
Let $\widetilde{\mathrm{C}}_{0}^{\infty}\left(\mathbb{R}^{3}\right)$ denote the set of smooth $S O(3)$-invariant functions with compact support, i.e.

$$
\widetilde{\mathrm{C}}_{0}^{\infty}\left(\mathbb{R}^{3}\right):=\left\{\phi \in \mathrm{C}_{0}^{\infty}\left(\mathbb{R}^{3}\right) \mid \phi=\rho_{a}^{*} \phi \text { for all } a \in S O(3)\right\} .
$$

In other words, $\widetilde{\mathrm{C}}_{0}^{\infty}\left(\mathbb{R}^{3}\right)$ is the set of radial functions on $\mathbb{R}^{3}$. We then define the space of static spherically symmetric Higgs fields with compact support by

$$
\mathcal{H}_{0}^{\infty}:=\left\{\phi(x) x^{j} \tau_{j} \mid \phi \in \widetilde{\mathrm{C}}_{0}^{\infty}\left(\mathbb{R}^{3}\right)\right\}
$$

where

$$
\tau_{1}=\frac{1}{2 i}\left(\begin{array}{cc}
0 & 1 \\
1 & 0
\end{array}\right), \tau_{2}=\frac{1}{2 i}\left(\begin{array}{cc}
0 & -i \\
i & 0
\end{array}\right), \tau_{3}=\frac{1}{2 i}\left(\begin{array}{cc}
1 & 0 \\
0 & -1
\end{array}\right),
$$

is a basis for $\mathfrak{s u}(2)$. We will choose the normalization of the Ad-invariant innerproduct $\langle\cdot \mid \cdot\rangle$ so that

$$
\left\langle\tau_{i} \mid \tau_{j}\right\rangle=\delta_{i j} .
$$

Completing $\mathcal{H}_{0}^{\infty}$ in the $\mathrm{W}_{\delta}^{k, p}\left(\mathbb{R}^{3}, \mathfrak{s u}(2)\right)$ norm gives

$$
\mathcal{H}_{\delta}^{k, p}:=\overline{\mathcal{H}_{0}^{\infty}} \subset \mathrm{W}_{\delta}^{k, p}\left(\mathbb{R}^{3}, \mathfrak{s u}(2)\right) .
$$

Proposition 4.2. Suppose $f \in C^{\infty}([0, \infty))$ satisfies $1-f(r)=O\left(r^{2}\right)$ as $r \rightarrow 0$, $f(r)=O\left(r^{-\eta}\right)$ as $r \rightarrow \infty$ for some $\eta>0$, and $f \geq 0$. Then for $1<p<\infty$, $-1<\delta<0$, and $k \in \mathbb{N}_{0}$ the operator

$$
\mathcal{H}_{\delta}^{k+2, p} \longrightarrow \mathcal{H}_{\delta-2}^{k, p}: \Phi(x) \longmapsto \Delta \Phi(x)+\frac{2}{|x|^{2}}(1-f(|x|)) \Phi(x)
$$

is an isomorphism.

Proof. Without loss of generality we can assume that $k=0$. We first show that the operator

$$
P:=\Delta+2|x|^{-2}(1-f(|x|))
$$

has a finite dimensional kernel and closed range on the space of static spherically symmetric Higgs fields.

Lemma 4.3. For $-1<\delta<0,1<p<\infty$ the operator $P$ defines a continuous map from $\mathcal{H}_{\delta}^{2, p} \rightarrow \mathcal{H}_{\delta-2}^{0, p}$ that has closed range and a finite dimensional kernel.

Proof. Directly from the definition of the weighted spaces it is easy to see that $P$ defines a continuous map from $\mathrm{W}_{\delta}^{2, p}\left(\mathbb{R}^{3}, \mathfrak{s u}(2)\right)$ to $\mathrm{W}_{\delta-2}^{0, p}\left(\mathbb{R}^{3}, \mathfrak{s u}(2)\right)$. A calculation shows that $P\left(\mathcal{H}_{0}^{\infty}\right) \subset \mathcal{H}_{0}^{\infty}$ and hence $P$ defines a continuous map from $\mathcal{H}_{\delta}^{2, p} \rightarrow$ $\mathcal{H}_{\delta-2}^{0, p}$.

Suppose $\Phi \in \mathcal{H}_{0}^{\infty}$. Then split $\Phi$ as $\Phi=\Phi_{0}+\Phi_{\infty}$ where $\Phi_{0}=\chi_{2} \Phi$ and $\Phi_{\infty}=\left(1-\chi_{2}\right) \Phi$. Since $\Phi(x)=\phi(x) x^{j} \tau_{j}$ for some $\phi \in \widetilde{\mathrm{C}}_{0}^{\infty}\left(\mathbb{R}^{3}\right)$,

$$
\Phi_{\infty}(x)=\frac{\phi_{\infty}(x)}{|x|} x^{j} \tau_{j}
$$


where $\phi_{\infty}(x):=|x|\left(1-\chi_{2}(x)\right) \phi(x) \in \widetilde{\mathrm{C}}_{0}^{\infty}\left(\mathbb{R}^{3}\right)$.

Straightforward calculation verifies that for $|x|>0,\left|\Phi_{\infty}\right|^{2}=\left|\phi_{\infty}\right|^{2},\left|\partial \Phi_{\infty}\right|^{2}=$ $\left|\partial \phi_{\infty}\right|^{2}+2|x|^{-2}\left|\phi_{\infty}\right|^{2}$ and $\left|\partial^{2} \Phi_{\infty}\right|^{2}=\left|\partial^{2} \phi_{\infty}\right|^{2}+8|x|^{2-}\left|\partial \phi_{\infty}\right|^{2}+6|x|^{-4}\left|\phi_{\infty}\right|^{2}$. Using this and supp $|\phi|, \operatorname{supp}|\Phi| \subset E_{1}(0)$ it follows that there exists a $C$ independent of $\phi$ and $\Phi$ such that

$$
\left\|\Phi_{\infty}\right\|_{2, p, \delta} \leq C\left\|\phi_{\infty}\right\|_{2, p, \delta} .
$$

A short calculation shows that

$$
P \Phi_{\infty}(x)=\left(\Delta \phi_{\infty}(x)-\frac{2 f(|x|)}{|x|^{2}} \phi_{\infty}(x)\right) \frac{x^{k}}{|x|} \tau_{k} .
$$

Thus if we define

$$
Q:=\Delta-\frac{2\left(1-\chi_{1 / 4}(x)\right) f(|x|)}{|x|^{2}},
$$

then $P \Phi_{\infty}(x)=Q \phi_{\infty}(x) \frac{x^{k}}{\mid x} \tau_{k}$ since supp $\left|\phi_{\infty}\right| \subset E_{0}$. So $\left|P \Phi_{\infty}(x)\right|=\left|Q \phi_{\infty}(x)\right|$ and hence

$$
\left\|P \Phi_{\infty}\right\|_{0, p, \delta-2}=\left\|Q \phi_{\infty}\right\|_{0, p, \delta-2} .
$$

In the terminology of [1], the operator $Q$ is asymptotic to $\Delta$. Therefore by [1] theorem 1.10 we have the estimate

$$
\left\|\phi_{\infty}\right\|_{2, p, \delta} \leq C\left(\left\|Q \phi_{\infty}\right\|_{0, p, \delta-2}+\left\|\phi_{\infty}\right\|_{p ; B_{R}(0)}\right)
$$

for some $R>0$. Since $\left\|\phi_{\infty}\right\|_{p ; B_{R}(0)}=\left\|\Phi_{\infty}\right\|_{p ; B_{R}(0)}$, we get the following estimate from (4.4), (4.6), and (4.7)

$$
\left\|\Phi_{\infty}\right\|_{2, p, \delta} \leq C\left(\left\|P \Phi_{\infty}\right\|_{0, p, \delta-2}+\left\|\Phi_{\infty}\right\|_{p ; B_{R}(0)}\right) .
$$

Once we have this scale broken estimate we can proceed as in the proof of theorem 1.10 of [1] to conclude that $P$ has closed range and a finite dimensional kernel.

With respect to the pairing $(\Psi, \Phi)=\int\langle\Psi \mid \Phi\rangle d^{3} x$ the operator has a formal adjoint $P^{*}=P$. Since $W_{\delta-2}^{0, p}\left(\mathbb{R}^{3}, \mathfrak{s u}(2)\right)^{*}=W_{-1-\delta}^{0, p^{\prime}}\left(\mathbb{R}^{3}, \mathfrak{s u}(2)\right)$ where $p^{\prime}=$ $p /(p-1)$, it follows from proposition 3.4 and proposition 1.14 of [1] that $\operatorname{ker} P^{*} \subset$ $W_{-1-\delta}^{2, p}\left(\mathbb{R}^{3}, \mathfrak{s u}(2)\right)$. Therefore by the above lemma

$$
\left.\operatorname{dim} \operatorname{coker} P\right|_{\mathcal{H}_{\delta}^{2, p}}=\left.\operatorname{dim} \operatorname{ker} P\right|_{\mathcal{H}_{-1-\delta}^{2, p}}<\infty \text {. }
$$

Lemma 4.4. For any $\delta<0,1<p<\infty$, $\left.\operatorname{ker} P\right|_{\mathcal{H}_{\delta}^{2, p}}=\{0\}$.

Proof. Suppose $\Psi \in \mathcal{H}_{\delta}^{2, p}$ satisfies $P \Psi=0$. Then by elliptic regularity (see [8] theorem 9.19 or [7] theorem 3.6), $P \Psi=0$ implies that $\Psi \in C^{\infty}\left(\mathbb{R}^{3}, \mathfrak{s u}(2)\right)$. So there exists a function $\psi(r) \in C^{\infty}([0, \infty))$ such that

$$
\Psi(x)=\psi(|x|) \frac{x^{j}}{|x|} \tau_{j} \quad \text { and } \quad \psi(r)=c r+\mathrm{O}\left(r^{3}\right) \quad \text { as } r \rightarrow 0 .
$$


It follows from the equality $|\Psi(x)|=|\psi(|x|)|$ and theorems 1.2 of [1] that $\psi(x)=$ $\mathrm{o}\left(|x|^{\delta}\right), \partial_{i} \psi(x)=\mathrm{o}\left(|x|^{\delta-1}\right)$, and $\partial_{i} \partial_{j} \psi(x)=\mathrm{o}\left(|x|^{\delta-2}\right)$ as $|x| \rightarrow \infty$. Now $P \Psi=0$ implies that (see (4.5D) $\Delta \psi(x)-|x|^{-2} 2 f(|x|) \psi(x)=0$. Multiplying by $\psi(x)$ yields $\psi \Delta \psi-2 f(|x|)|x|^{-2} \psi^{2}=0$ which by the fall off conditions for $\psi$ near $|x|=0$ and $|x|=\infty$ is integrable. Integrating yields

$$
\int_{\mathbb{R}^{3}} \psi \Delta \psi d^{3} x-\int_{\mathbb{R}^{3}} \frac{2 f(|x|)}{|x|^{2}} \psi^{2} d^{3} x=0 .
$$

Integrating by parts which is again valid by the fall off conditions conditions then gives

$$
\int_{\mathbb{R}^{3}}|\partial \psi|^{2} d^{3} x+\int_{\mathbb{R}^{3}} \frac{2 f(|x|)}{|x|^{2}} \psi^{2} d^{3} x=0 .
$$

Thus $f \geq 0$ implies that $\psi=0$ and hence $\Psi=0$.

The proof now follows from (4.9) and lemmas 4.3 and 4.4 which imply that $\left.\operatorname{dim} \operatorname{coker} P\right|_{\mathcal{H}_{\delta}^{2, p}}=\left.\operatorname{dim} \operatorname{ker} P\right|_{\mathcal{H}_{\delta}^{2, p}}=0$.

In addition to being spherically symmetric, we will assume that our gauge potential is purely magnetic. Choosing an appropriate gauge, the gauge potential can then be written as $[2]$

$$
A_{0}=0 \quad \text { and } \quad A_{i}(x):=a(|x|) \epsilon_{i}{ }_{k}{ }_{k} x^{k} \tau_{j} .
$$

If we write the gauge potential $A_{i}$ as a 3 -tuple $A=\left(A_{1}, A_{2}, A_{3}\right)$ then the gauge potential $A$ takes values in the space $\mathfrak{s u}(2)^{3}$ which carries a norm

$$
|A|^{2}:=\sum_{i=1}^{3}\left\langle A_{i} \mid A_{i}\right\rangle
$$

We define the set of smooth static spherically symmetric purely magnetic gauge potentials with compact support by

$$
\mathcal{A}_{0}^{\infty}:=\left\{A: \mathbb{R}^{3} \rightarrow \mathfrak{s u}(2)^{3} \mid A_{i}(x)=a(x) \epsilon_{i}{ }^{j}{ }_{k} x^{k} \tau_{j} \text { for some } a \in \widetilde{\mathrm{C}}_{0}^{\infty}\left(\mathbb{R}^{3}\right)\right\} .
$$

Completing this in the $\mathcal{W}_{\mu}^{k, p}\left(\mathbb{R}^{3}, \mathfrak{s u}(2)^{3}\right)$ gives

$$
\mathcal{A}_{\mu}^{k, p}=\overline{\mathcal{A}_{0}^{\infty}} \subset \mathcal{W}_{\mu}^{k, p}\left(\mathbb{R}^{3}, \mathfrak{s u}(2)^{3}\right) .
$$

Notice that every $A \in \mathcal{A}_{0}^{\infty}$ satisfies

$$
\operatorname{div} A:=\sum_{j=1}^{3} \partial_{j} A_{j}=0,
$$

which implies by the continuity of differentiation (see (3.5)) that

$$
\operatorname{div} A=0 \text { for all } A \in \mathcal{A}_{\mu}^{k, p} .
$$


This shows that the static spherically symmetric gauge potentials satisfy the Coulomb gauge condition globally on $\mathbb{R}^{3}$. As is well known, this is a very special situation and is one of the reasons that makes the static spherically symmetric Yang-Mills equations easy to analyze.

Proposition 4.5. For $\kappa-|\mu|>0, k \in \mathbb{N}_{0}$ and $1<p<\infty$, the operator $\Delta-\kappa^{2}$ : $\mathcal{A}_{\mu}^{k+2, p} \rightarrow \mathcal{A}_{\mu}^{k, p}$ is an isomorphism.

Proof. Follows from directly from theorem 3.12 using the same arguments as in the proof of proposition 4.1.

\section{The modified Yang-Mills equation}

Instead of solving the Yang-Mills equation (2.25) we will instead solve a related system of equations whose solutions will also be solutions to (2.25). The reason for this modification is to make the Yang-Mills equation differentiable on the spherically symmetric function spaces introduced in section 4.

We begin by splitting the YM potential and the Higgs fields. Let

$$
Y=Y_{j} d x^{j}=\frac{\chi_{1}(x)-1}{r^{2}} \epsilon_{i}{ }_{k} x^{k} \tau_{j} \quad, \quad \Omega=\frac{1-\chi_{1}(x)}{r} x^{j} \tau_{j} .
$$

and

$$
A=Y+Z, \quad \Phi=\Omega+\Psi
$$

where $Z \in \mathcal{A}_{\mu}^{k, p}$ and $\Psi \in \mathcal{H}_{\delta}^{k, p}$ will be considered as the unknowns. Assume for the moment that $Z$ and $\Psi$ are $C^{1}$ and spherically symmetric. Then we can write

$$
Z_{j}=z(r) \epsilon_{i}{ }_{k} x^{k} \tau_{j} \quad \text { and } \quad \Psi=\psi(r) x^{j} \tau_{j}
$$

and a short calculation shows that $\left[\Phi, D_{0} \Phi\right]=0$ and

$$
\left[\Phi, D_{i} \Phi\right]=\left(\frac{1-\chi_{1}}{r}+\psi\right)^{2}\left(\chi_{1}+r^{2} z\right) \epsilon_{i}{ }^{j}{ }_{k} x^{k} \tau_{j}=|\Omega+\Psi|^{2}\left(\frac{\chi_{1}}{r^{2}} \epsilon_{i}{ }_{k} x^{k} \tau_{j}+Z_{i}\right) .
$$

Thus for $C^{1}$ static spherically symmetric fields we have the identity

$$
\left[\Phi, D_{\alpha} \Phi\right]=\left(1-\delta_{\alpha}^{0}\right)\left(\chi_{1}\left[\Phi, D_{\alpha} \Phi\right]+\left(1-\chi_{1}\right)|\Phi|^{2}\left(\frac{\chi_{1}}{r^{2}} \delta_{\alpha}^{i} \epsilon_{i}{ }^{j}{ }_{k} x^{k} \tau_{j}+\delta_{\alpha}^{i} Z_{i}\right)\right) .
$$

This motivates us to consider the following modified Yang-Mills equation

$$
\begin{gathered}
\overline{\mathfrak{g}}^{\alpha \nu}\left(\left(1-\chi_{3}\right) D_{\alpha}^{Y} F_{\nu \beta}^{Y}+D_{\alpha}^{A} F_{\nu \beta}^{A}\right) \\
-\left(1-\delta_{\beta}^{0}\right)\left(\chi_{1}\left[\Phi, D_{\beta} \Phi\right]+\left(1-\chi_{1}\right)|\Omega+\Psi|^{2}\left(\frac{\chi_{1}}{r^{2}} \delta_{\beta}^{i} \epsilon_{i}{ }^{j}{ }_{k} x^{k} \tau_{j}+Z_{\beta}\right)\right)=0
\end{gathered}
$$

where $A=Y+Z$ and $\Phi=\Psi+\Omega$. Observe that if the term $\left(1-\chi_{3}\right) D_{\alpha}^{Y} F_{\nu \beta}^{Y}$ vanished then this equation would be the same as equation (2.25) modified by the 
identity (5.2) and written in term of the new variables $Z$ and $\Psi$. We shall see later that for static spherically symmetric solutions the $\left(1-\chi_{3}\right) D_{\alpha}^{Y} F_{\nu \beta}^{Y}$ does vanish. This will show that solutions to (5.3) will be solutions to (2.25). Our assumption that the fields are static and spherically symmetric imply that $F_{\alpha 0}^{A}=0, F_{\alpha 0}^{Y}=0$ and $\Gamma_{l 0}^{k}=0$ and hence equation (5.3) will be satisfied automatically for $\beta=0$. Therefore we need only solve

$$
\begin{gathered}
\overline{\mathfrak{g}}^{\alpha \nu}\left(\left(1-\chi_{3}\right) D_{\alpha}^{Y} F_{\nu i}^{Y}+D_{\alpha}^{A} F_{\nu i}^{A}\right)- \\
\left(\chi_{1}\left[\Phi, D_{i} \Phi\right]+\left(1-\chi_{1}\right)|\Phi|^{2}\left(\frac{\chi_{1}}{r^{2}} \epsilon_{i}{ }^{j}{ }_{k} x^{k} \tau_{j}+Z_{i}\right)\right)=0 .
\end{gathered}
$$

In terms of the new variables $Z$ and $\Psi$ the Higgs equations (2.26) becomes

$$
\begin{gathered}
\overline{\mathfrak{g}}^{\alpha \beta}\left(\partial_{\alpha} D_{\beta}^{Y} \Omega-\Gamma_{\alpha \beta}^{\sigma} D_{\sigma}^{Y} \Omega+\left[Y_{\alpha}, D_{\beta}^{Y} \Omega\right]+\partial_{\alpha} D_{\beta}^{Y} \Psi-\Gamma_{\alpha \beta}^{\sigma} D_{\sigma}^{Y} \Psi+\left[Y_{\alpha}, D_{\beta}^{Y} \Psi\right]+\right. \\
\partial_{\alpha}\left[Z_{\beta}, \Omega+\Psi\right]-\Gamma_{\alpha \beta}^{\sigma}\left[Z_{\sigma}, \Omega+\Psi\right]+\left[Y_{\alpha},\left[Z_{\beta}, \Omega+\Psi\right]\right]+ \\
\left.\left[Z_{\alpha}, D_{\beta}^{Y} \Omega+D_{\beta}^{Y} \Psi+\left[Z_{\beta}, \Omega+\Psi\right]\right]\right)=0
\end{gathered}
$$

\section{Analyticity of the field equations}

In this section we establish that the reduced field equations (2.18) and the modified YMH equations (5.4)-(5.5) define analytic maps. For a definition of analytic maps between Banach spaces see [6] definition 15.1. As is standard we will use $\mathrm{C}^{\omega}$ to denote the class of analytic maps. To establish analyticity we will repeatedly use the following: continuous linear and bilinear maps between Banach spaces are analytic, and the composition of two analytic maps is again analytic. Also useful is proposition 3.6 of [9] which shows how analytic functions on $\mathbb{R}$ can be used to define analytic maps on Banach algebras.

To begin we first fix some notation. If $V$ is a Banach space with norm $\|\cdot\|$ then we define $B_{V}(x ; R)$ to be the ball of radius $R$ centered at $x \in V$. We recall the following results from [9] which are fundamental in establishing analyticity.

Proposition 6.1. [Proposition 3.10, [9]] Suppose $3 / 2<p<\infty$ and $-1<\delta<0$. Then for any $R>0$ there exists a $\Lambda>0$ such that the following maps are of class $C^{\omega}$ :

$$
\begin{aligned}
& (-\Lambda, \Lambda) \times B_{W_{\delta}^{2, p}\left(\mathbb{R}^{3}, \mathbb{S}\right)}(0 ; R) \rightarrow W_{\delta}^{2, p}\left(\mathbb{R}^{3}, \mathbb{S}\right):(\lambda, \mathfrak{U}) \mapsto\left(\overline{\mathfrak{g}}^{\alpha \beta}-\overline{\mathfrak{g}}_{o}^{\alpha \beta}\right) \\
& (-\Lambda, \Lambda) \times B_{W_{\delta}^{k, p}\left(\mathbb{R}^{3}, \mathbb{S}\right)}(0 ; R) \rightarrow W_{\delta}^{2, p}\left(\mathbb{R}^{3}, \mathbb{S}\right):(\lambda, \mathfrak{U}) \mapsto\left(\overline{\mathfrak{g}}_{\alpha \beta}-\overline{\mathfrak{g}}_{o \beta}\right)
\end{aligned}
$$

and

$$
(-\Lambda, \Lambda) \times B_{W_{\delta}^{2, p}\left(\mathbb{R}^{3}, \mathbb{S}\right)}(0 ; R) \rightarrow W_{\delta}^{2, p}\left(\mathbb{R}^{3}\right):(\lambda, \mathfrak{U}) \mapsto|\mathfrak{d}|^{q / 2}-1
$$


for $q=-3,-2,-1,1,2$. Moreover, the following expansions are valid

$$
\begin{gathered}
|\mathfrak{d}|-1=-4 \lambda \mathfrak{U}^{00}+O\left(\lambda^{2}\right), \quad \sqrt{\mathfrak{d}}-1=-2 \lambda \mathfrak{U}^{00}+O\left(\lambda^{2}\right), \\
\frac{1}{\sqrt{\mathfrak{d}}}-1=2 \lambda \mathfrak{U}^{00}+O\left(\lambda^{2}\right), \quad\left(\overline{\mathfrak{g}}_{\alpha \beta}-\overline{\mathfrak{g}}_{\alpha \beta}\right)=-4 \lambda\left(\delta_{\alpha}^{0} \delta_{\beta}^{0}\right) \mathfrak{U}^{00}+O\left(\lambda^{2}\right) .
\end{gathered}
$$

Proposition 6.2. [Proposition 6.2, [9]] Suppose $p>3$ and $-1<\delta<0$. Then for any $R>0$ there exists a $\Lambda>0$ such that the Christofel symbols

$$
\Gamma_{\beta \gamma}^{\alpha}:(-\Lambda, \Lambda) \times B_{W_{\delta}^{2, p}\left(\mathbb{R}^{3}, \mathbb{S}\right)}(0 ; R) \rightarrow W_{\delta-1}^{1, p}\left(\mathbb{R}^{3}\right)
$$

are of class $C^{\omega}$ for all $\alpha, \beta, \gamma=0,1,2,3$. Moreover, the following expansion is valid

$$
\Gamma_{\beta \gamma}^{\alpha}=\left.\Gamma_{\beta \gamma}^{\alpha}\right|_{\lambda=0}+O(\lambda)
$$

where

$$
\left.\Gamma_{\beta \gamma}^{\alpha}\right|_{\lambda=0}=\left\{\begin{array}{ll}
\mathfrak{U}^{00}, \alpha & \text { if } \beta=\gamma=0 \text { and } \alpha \neq 0 \\
0 & \text { otherwise }
\end{array} .\right.
$$

It is important to note that

$$
\left.\underset{o}{\left(\overline{\mathfrak{g}}^{\alpha \beta}\right.}\right)=\left(\begin{array}{cccc}
-\lambda & 0 & 0 & 0 \\
0 & 1 & 0 & 0 \\
0 & 0 & 1 & 0 \\
0 & 0 & 0 & 1
\end{array}\right)
$$

so that

$$
\left.\underset{o}{\left(\overline{\mathfrak{g}}^{\alpha \beta}\right)}\right|_{\lambda=0}=\left(\begin{array}{cccc}
0 & 0 & 0 & 0 \\
0 & 1 & 0 & 0 \\
0 & 0 & 1 & 0 \\
0 & 0 & 0 & 1
\end{array}\right)
$$

Using the above propositions and the results from section 3 we can establish that the stress-energy tensor defines an analytic map.

Proposition 6.3. Suppose $p>3,-1<\delta<0$ and $\mu<0$. Then for any $R>0$ there exists a $\Lambda>0$ such that

$T:(-\Lambda, \Lambda) \times B_{\mathcal{U}_{\delta}^{2, p}}(0 ; R) \times \mathcal{H}_{\delta}^{2, p} \times \mathcal{A}_{\mu}^{2, p} \longrightarrow W_{\delta-2}^{1, p}\left(\mathbb{R}^{3}, \mathbb{S}\right):(\lambda, \mathfrak{U}, \Psi, Z) \longmapsto\left(T^{\alpha \beta}\right)$

and

$\mathcal{T}:(-\Lambda, \Lambda) \times B_{\mathcal{U}_{\delta}^{2, p}}(0 ; R) \times \mathcal{H}_{\delta}^{2, p} \times \mathcal{A}_{\mu}^{2, p} \longrightarrow W_{\delta-2}^{1, p}\left(\mathbb{R}^{3}, \mathbb{S}\right):(\lambda, \mathfrak{U}, \Phi, Z) \longmapsto\left(\mathcal{T}^{\alpha \beta}\right)$

are of class $C^{\omega}$. Moreover, the following expansion is valid

$$
\mathcal{T}^{0 \alpha}=\mathcal{T}^{\alpha 0}=O(\lambda) \quad \text { and } \quad \mathcal{T}^{i j}=4 \pi G T_{o}^{i j}(\Psi, Z)+O(\lambda)
$$


where

$$
\begin{aligned}
& T_{o}^{i j}(\Psi, Z)=\left(\delta^{i k} \delta^{j l}\left\langle D_{o}^{A} \Phi \mid D_{o}^{A} \Phi\right\rangle-\frac{1}{2} \delta^{i j} \delta^{k l}\left\langle D_{o}^{A} \Phi \mid D_{o}^{A} \Phi\right\rangle\right)+ \\
& \left(\delta^{i k} \delta^{j l} \delta^{m n}\left\langle F_{k m}^{A} \mid F_{l n}^{A}\right\rangle-\frac{1}{4} \delta^{k l} \delta^{m n} \delta^{i j}\left\langle F_{k m}^{A} \mid F_{l n}^{A}\right\rangle\right),
\end{aligned}
$$

and $A=Y+Z$ and $\Phi=\Omega+\Psi$.

Proof. Letting $A=Y+Z$, we can write

$$
F_{\alpha \beta}^{A}=F_{\alpha \beta}^{Y}+F_{\alpha \beta}^{Z}+\left[Y_{\alpha}, Z_{\beta}\right]+\left[Z_{\alpha}, Y_{\beta}\right]
$$

where

$$
F_{0 \alpha}^{Y}=0, \quad F_{i j}^{Y}=\epsilon_{i j k}\left[\frac{\chi_{1}^{\prime}(r)}{r}\left(\delta^{k l}-\frac{x^{k} x^{l}}{r^{2}}\right)+\frac{\chi_{1}(r)^{2}-1}{r^{4}} x^{k} x^{l}\right] \tau_{l}
$$

and $(\cdot)^{\prime}=\frac{d}{d r}(\cdot)$. Since $-1<\delta<0$, we get from (6.4) that

$$
F_{\alpha \beta}^{Y} \in \mathrm{W}_{\delta-1}^{2, p}\left(\mathbb{R}^{3}\right) .
$$

From the definition of $Y$ it is clear that

$$
Y \in \mathcal{A}_{-1}^{0, \infty} \text {. }
$$

Then since $\mu<0$ and $p>3$, it follows from the inclusion (3.7), the multiplication lemma 3.3 the weighted Hölder inequality (theorem 1.2 (ii), [1]), and equations (6.3), (6.5), (6.6) that the map

$$
\mathcal{A}_{\mu}^{2, p} \longrightarrow \mathrm{W}_{\delta-1}^{1, p}: Z \longmapsto F^{A} \text { is analytic. }
$$

Also note that for $\Psi \in \mathrm{W}^{k, p}$, (6.6) implies via the weighted Hölder inequality (theorem 1.2 (ii), [1]) that $\left[Y_{\alpha}, \Psi\right] \in \mathrm{W}_{\delta-1}^{2, p}$. There for the map

$$
D_{\alpha}^{Y}: \mathrm{W}_{\delta}^{2, p}\left(\mathbb{R}^{3}, \mathfrak{s u}(2)\right) \longrightarrow \mathrm{W}_{\delta-1}^{1, p}\left(\mathbb{R}^{3}\right): \Psi \longmapsto \partial_{\alpha} \Psi+\left[Y_{\alpha}, \Psi\right]
$$

is continuous. A short calculation shows that

$$
\begin{aligned}
& D_{0}^{Y} \Omega=0, \\
& D_{i}^{Y} \Omega=\left(-\frac{\chi_{1}^{\prime}(r)}{r^{2}}-\frac{\left(1-\chi_{1}(r)\right)}{r^{3}} \chi_{1}(r)\right) x^{i} x^{k} \tau_{k}+\frac{1-\chi_{1}(r)}{r} \chi_{1}(r) \tau_{i},
\end{aligned}
$$

and

$$
D_{\alpha}^{A} \Phi=D_{\alpha}^{Y} \Omega+D_{\alpha}^{Y} \Psi+\left[Z_{\alpha}, \Omega+\Psi\right]
$$

Again, because $\mu<0$ and $p>3$, the inclusion (3.7), the multiplication lemma 3.3 and (6.8), (6.9), (6.10), (6.11) imply that

$$
\mathrm{W}_{\delta}^{2, p}\left(\mathbb{R}^{3}, \mathfrak{s u}(2)\right) \times \mathcal{A}_{\mu}^{2, p} \longrightarrow \mathrm{W}_{\delta-1}^{1, p}\left(\mathbb{R}^{3}\right):(\Psi, Z) \longmapsto D_{\alpha}^{A} \Phi
$$

is analytic. The analyticity of the maps now follows from lemma 3.3 proposition 6.1, (6.7), and (6.12). 
Letting

$$
\begin{aligned}
& { }_{1} \Xi^{\alpha \beta}=E^{\alpha \beta}-\mathcal{T}^{\alpha \beta} \\
& { }_{2} \Xi=\overline{\mathfrak{g}}^{\alpha \beta} D_{\alpha}^{A} D_{\beta}^{A} \Phi \\
& \left.{ }_{3} \Xi_{i}=\overline{\mathfrak{g}}^{\alpha \nu}\left(\chi_{3}-1\right) D_{\alpha}^{Y} F_{\nu i}^{Y}+D_{\alpha}^{A} F_{\nu i}^{A}\right)-\left(\chi_{1}\left[\Phi, D_{j} \Phi\right]+\right. \\
& \left.\quad\left(1-\chi_{1}\right)|\Phi|^{2}\left(\frac{\chi_{1}}{r^{2}} \epsilon_{i}{ }_{k}{ }_{k} x^{k} \tau_{j}+Z_{i}\right)\right)
\end{aligned}
$$

and

$$
\Xi=\left({ }_{1} \Xi,{ }_{2} \Xi,{ }_{3} \Xi\right)=\left(\left({ }_{1} \Xi^{\alpha \beta}\right),{ }_{2} \Xi,\left({ }_{3} \Xi_{i}\right)\right) .
$$

we collect our field equations (2.18), (5.4), and (5.5) into a single expression

$$
\Xi=0 \text {. }
$$

Proposition 6.4. Suppose $p>3,-1<\delta<-3 / p$ and $\mu<0$ and

$$
X=W_{\delta-2}^{0, p}\left(\mathbb{R}^{3}, \mathbb{S}\right) \times W_{\delta-2}^{0, p}\left(\mathbb{R}^{3}, \mathfrak{s u}(2)\right) \times \mathcal{W}_{\mu}^{0, p}\left(\mathbb{R}^{3}, \mathfrak{s u}(2)^{3}\right) .
$$

Then for any $R>0$ there exists a $\Lambda>0$ such that

$\left.\Xi:(-\Lambda, \Lambda) \times B_{\mathfrak{U}_{\delta}^{2, p}}(0 ; R) \times \mathcal{H}_{\delta}^{2, p} \times \mathcal{A}_{\mu}^{2, p} \longrightarrow X:(\lambda, \mathfrak{U}, \Psi, Z) \longmapsto\left(\left({ }_{1} \Xi^{\alpha \beta}\right),{ }_{2} \Xi,{ }_{3} \Xi_{j}\right)\right)$

is of class $C^{\omega}$. Moreover the following expansions are valid

$$
\begin{aligned}
& { }_{1} \Xi^{0 \alpha}=\Delta \mathfrak{U}^{0 \alpha}+O(\lambda), \\
& { }_{1} \Xi^{i j}=\Delta \mathfrak{U}^{i j}-\delta^{i k} \delta^{j l} \partial_{k} \mathfrak{U}^{00} \partial_{l} \mathfrak{U}^{00}+\frac{1}{2} \delta^{i j} \delta^{k l} \partial_{k} \mathfrak{U}^{00} \partial_{l} \mathfrak{U}^{00}-4 \pi G T_{o}^{i j}+O(\lambda), \\
& { }_{2} \Xi=\delta^{i j}{ }_{o}^{A} D_{o}^{A} \Phi+O(\lambda), \\
& { }_{3} \Xi_{i}=\delta^{k l}{ }_{o}{ }_{o}^{A} F_{l i}^{A}-\left(\chi_{1}\left[\Phi, D_{o}{ }_{i}^{A} \Phi\right]+\left(1-\chi_{1}\right)|\Phi|^{2}\left(\frac{\chi_{1}}{r^{2}} \epsilon_{i}{ }_{k}{ }_{k} x^{k} \tau_{j}+Z_{i}\right)\right)+O(\lambda) .
\end{aligned}
$$

where $A=Y+Z$ and $\Phi=\Omega+\Psi$.

Proof. This proposition can be proved in a similar manner to the proof of proposition 6.3 by using the inclusions (3.4), (3.6), and (3.7), the two multiplication lemmas 3.3 and 3.10, theorem 3.7 and [1] theorem 1.2, and propositions 6.1, 6.2, and 6.3. Note that that formulas used in the proof of proposition 6.3 are also useful.

The expansion in $\lambda$ can be inferred from (6.1) and (6.2), the expansions in propositions 6.1, 6.2, and 6.3, and

$$
\left[\left(1-\chi_{3}\right) \overline{\mathfrak{g}}^{\alpha \nu} D_{\alpha}^{Y} F_{\nu i}^{Y}\right]_{\lambda=0}=\left(1-\chi_{3}\right) \delta^{j k} D_{o}^{Y} F_{k i}^{Y}=0 .
$$


The last equality can be seen from

$$
\begin{aligned}
\delta^{j k} D_{o}^{Y} F_{k i}^{Y} d x^{i}= & \left(\chi_{1}^{\prime \prime}-\frac{\left(\chi_{1}^{2}-1\right) \chi_{1}}{r^{2}}\right)\left(-\sin \phi \tau_{1}+\cos \phi \tau_{2}\right) d \theta \\
& +\left(\chi_{1}^{\prime \prime}-\frac{\left(\chi_{1}^{2}-1\right) \chi_{1}}{r^{2}}\right)\left(\tau_{3}-\cot \theta\left(\sin \phi \tau_{2}+\cos \phi \tau_{1}\right)\right) d \phi
\end{aligned}
$$

where $(\cdot)^{\prime}=\frac{d}{d r}(\cdot)$.

Proposition 6.5. Suppose $p>3,-1<\delta<-3 / p$ and $\mu<0$. Then for any $R>0$ there exists a $\Lambda>0$ such that

$$
\begin{gathered}
\Xi:(-\Lambda, \Lambda) \times B_{\mathfrak{U}_{\delta}^{2, p}}(0 ; R) \times \mathcal{H}_{\delta}^{2, p} \times \mathcal{A}_{\mu}^{2, p} \longrightarrow \mathcal{U}_{\delta-2}^{0, p} \times \mathcal{H}_{\delta-2}^{0, p} \times \mathcal{A}_{\mu}^{0, p} \\
\left.:(\lambda, \mathfrak{U}, \Psi, Z) \longmapsto\left(\left({ }_{1} \Xi^{\alpha \beta}\right),{ }_{2} \Xi,{ }_{3} \Xi_{j}\right)\right)
\end{gathered}
$$

is of class $C^{\omega}$.

Proof. For fixed $R$ let $\Lambda$ be as given by proposition 6.4. Then it can be shown by straightforward calculation that $\lambda \in(-\Lambda, \Lambda), \mathfrak{U} \in \mathcal{U}_{0}^{\infty} \cap B_{\mathcal{U}^{2, p}}(0 ; R), \Psi \in$ $\mathcal{H}_{0}^{\infty} \cap \mathcal{H}_{\delta}^{2, p}$, and $Z \in \mathcal{A}_{0}^{\infty} \cap \mathcal{A}_{\mu}^{2, p}$ implies that $\Xi(\lambda, \mathfrak{U}, \Psi, Z) \in \mathcal{U}^{\infty} \times \mathcal{H}^{\infty} \times \mathcal{A}^{\infty}$. The result now follows from the continuity of the map $\Xi$ (see proposition 6.4) and the density of $\mathcal{U}_{0}^{\infty}, \mathcal{H}_{0}^{\infty}$, and $\mathcal{A}_{0}^{\infty}$.

\section{Solving the reduced/modified EYMH equations}

We now employ the same method as in [9] to find solutions to the reduced/modified EYMH equations. Namely, we first solve the reduced equations for $\lambda=0$ and then use the implicit function theorem to show that there exists a solution for small $\lambda$.

$7.1 \lambda=0$

Fix $R>0$, assume $p>3,-1<\delta<-p / 3, \mu>0$, and let $\Lambda>0$ be as in proposition 6.5. Then the expansion from proposition 6.4 shows that

$$
\Xi(0, \mathfrak{U}, \Psi, Z)=0
$$

if and only if

$$
\begin{aligned}
& \Delta \mathfrak{U}^{0 \alpha}=0, \\
& \Delta \mathfrak{U}^{i j}=\delta^{i k} \delta^{j l} \partial_{k} \mathfrak{U}^{00} \partial_{l} \mathfrak{U}^{00}-\frac{1}{2} \delta^{i j} \delta^{k l} \partial_{k} \mathfrak{U}^{00} \partial_{l} \mathfrak{U}^{00}+4 \pi G T_{o}^{i j}(\Psi, Z), \\
& \delta^{i j} D_{i}^{A} D_{o}^{A} \Phi=0 \\
& \delta^{k l}{ }_{o}{ }_{k}^{A} F_{l i}^{A}-\left(\chi_{1}\left[\Phi, D_{o}^{A} \Phi\right]+\left(1-\chi_{1}\right)|\Phi|^{2}\left(\frac{\chi_{1}}{r^{2}} \epsilon_{i}{ }^{j}{ }_{k} x^{k} \tau_{j}+Z_{i}\right)\right),
\end{aligned}
$$


where $A=Y+Z$ and $\Phi=\Omega+\Psi$. Equations (7.1)- (7.4) can be regarded as the Newtonian YMH equations with $\mathfrak{U}^{00}$ playing the role of the Newtonian potential.

The BPS monopole solution to the Yang-Mills-Higgs equation is

$$
A^{b}=\frac{w-1}{r^{2}} \epsilon_{i}{ }_{k} x^{k} \tau_{j} \quad \text { and } \quad \Phi^{b}=\frac{\phi(r)}{r} x^{j} \tau_{j}
$$

where

$$
w(r)=\frac{r}{\sinh (r)} \quad \text { and } \quad \phi(r)=\operatorname{coth}(r)-\frac{1}{r} .
$$

From this we define

$$
Z^{b}:=A^{b}-Y=\frac{w-\chi_{1}}{r^{2}} \epsilon_{i}{ }_{k} x^{k} \tau_{j} i, \quad \Psi^{b}:=\Phi^{b}-\Omega=\frac{(\phi(r)-1)+\chi_{1}}{r} x^{j} \tau_{j},
$$

and also observe that

$$
Z^{b} \in \mathcal{A}_{\mu}^{k, p} \quad \text { and } \quad \Psi^{b} \in \mathcal{H}_{\delta}^{k, p} \quad \text { for } 1 \leq p \leq \infty, k \in \mathbb{N}_{0} \text { and } \delta, \mu>-1 .
$$

It can be checked that $\left(\Psi^{b}, Z^{b}\right)$ solve equations (7.3) and (7.4). Then using lemma (4.1),

$$
\mathfrak{U}_{b}^{0 \alpha}:=0, \quad \mathfrak{U}_{b}^{i j}:=\Delta^{-1} T_{o}^{i j}\left(\Psi^{b}, Z^{b}\right)
$$

solve the remaining equations (7.1) and (7.2) .

\section{$7.2 \lambda>0$}

To use the implicit function theorem, we first need to establish that the derivative of the map

$$
\Xi_{0}(\mathfrak{U}, \Psi, Z):=\Xi(0, \mathfrak{U}, \Psi, Z)
$$

evaluated at $\left(\mathfrak{U}_{b}, \Psi^{b}, Z^{b}\right)$ is an isomorphism.

Proposition 7.1. Suppose $p>6,-1<\delta<-3 / p$ and $-1<\mu<0$. Then the linear map

$$
D \Xi_{0}\left(\mathfrak{U}_{b}, \Psi^{b}, Z^{b}\right): \mathfrak{U}_{\delta}^{2, p} \times \mathcal{H}_{\delta}^{2, p} \times \mathcal{A}_{\mu}^{2, p} \longrightarrow \mathfrak{U}_{\delta-2}^{0, p} \times \mathcal{H}_{\delta-2}^{0, p} \times \mathcal{A}_{\mu}^{0, p}
$$

is an isomorphism.

Proof. For $\delta \Psi \in \mathcal{H}_{0}^{\infty}$, a short calculation shows that

$$
\delta^{i j}\left[A_{i}^{b},\left[A_{j}^{b}, \delta \Psi\right]\right]=-2 \frac{(w-1)^{2}}{r^{2}} \delta \Psi \quad \text { and } \quad \delta^{i j}\left[A_{i}^{b}, \partial_{j} \delta \Psi\right]=-2 \frac{(w-1)}{r^{2}} \delta \Psi .
$$

This and (4.10) shows that

$$
\delta^{i j} D_{o}^{A^{b}} D_{o}^{A^{b}} \delta \Psi=\Delta \delta \Psi-2 \frac{w^{2}(r)-1}{r^{2}} \delta \Psi \quad \text { for all } \delta \Psi \in \mathcal{H}_{0}^{\infty} .
$$


But since $\mathcal{H}_{0}^{\infty} \times \mathcal{A}_{0}^{\infty}$ is dense in $\mathcal{H}_{\delta}^{2, p} \times \mathcal{A}_{\mu}^{2, p}$, the continuity of the maps $\delta^{i j} D_{o}^{A^{b}} D_{o}^{A^{b}}$ : $\mathcal{H}_{0}^{2, p} \rightarrow \mathcal{H}_{\delta-2}^{0, p}($ see (6.8) $)$ and $\Delta-2 r^{-2}\left(w^{2}(r)-1\right): \mathcal{H}_{0}^{2, p} \rightarrow \mathcal{H}_{\delta-2}^{0, p}$ (see proposition (4.2) implies that

$$
\delta^{i j} D_{o}^{A^{b}}{ }_{o}^{A_{j}^{b}} \delta \Psi=\Delta \delta \Psi-2 \frac{w^{2}(r)-1}{r^{2}} \delta \Psi \quad \text { for all } \delta \Psi \in \mathcal{H}_{\delta}^{2, p} .
$$

Using this and (4.10), the derivative of $\Xi_{0}$ at $\left(\mathfrak{U}^{b}, \Psi^{b}, Z^{b}\right)$ can be written as

$$
\begin{aligned}
D \Xi\left(0, \Psi^{b}, Z^{b}\right) \cdot\left(\begin{array}{l}
\delta \mathfrak{U} \\
\delta \Psi \\
\delta Z
\end{array}\right)= & \left(\begin{array}{ccc}
\Delta & 0 & 0 \\
0 & \Delta-2 \frac{w^{2}-1}{r^{2}} & 0 \\
0 & 0 & \Delta-1
\end{array}\right)\left(\begin{array}{l}
\delta \mathfrak{U} \\
\delta \Psi \\
\delta Z
\end{array}\right) \\
& +\left(\begin{array}{ccc}
0 & J_{1} & J_{2} \\
0 & 0 & K_{12} \\
0 & K_{21} & K_{22}
\end{array}\right)\left(\begin{array}{l}
\delta \mathfrak{U} \\
\delta \Psi \\
\delta Z
\end{array}\right),
\end{aligned}
$$

where

$$
\begin{aligned}
& J_{1}(\delta \Psi)^{i j}=2 \delta^{i k} \delta^{j l}\left\langle D_{o}^{A^{b}} \Phi^{b} \mid{ }_{o}^{A^{b}} \delta \Psi\right\rangle-\delta^{i j} \delta^{k l}\left\langle{\underset{o}{k}}_{k}^{A^{b}} \Phi^{b} \mid{ }_{o}^{A^{b}} \delta \Psi\right\rangle, \\
& J_{2}(\delta Z)^{i j}=\left\langle D_{o}^{A^{b}} \Phi^{b} \mid\left[\delta Z_{l}, \Phi^{b}\right]\right\rangle-\delta^{i j} \delta^{k l}\left\langle D_{o}^{A^{b}} \Phi^{b} \mid\left[\delta Z_{l}, \Phi^{b}\right]\right\rangle+ \\
& 2\left\langle F_{k m}^{A^{b}} \mid \delta F_{l n}\right\rangle-\frac{1}{2} \delta^{k l} \delta^{m n} \delta^{i j}\left\langle F_{k m}^{A^{b}} \mid \delta F_{l n}\right\rangle, \\
& K_{12}(\delta Z)=\delta^{i j}\left(\partial_{i}\left[\delta Z_{j}, \Phi^{b}\right]+\left[\delta Z_{i}, D_{o}^{A^{b}} \Phi^{b}\right]+\left[A_{i}^{b},\left[\delta Z_{j}, \Phi^{b}\right]\right]\right), \\
& K_{21}(\delta \Psi)_{i}=-\chi_{1}\left(\left[\delta \Psi, D_{o}^{A^{b}} \Phi^{b}\right]+\left[\Phi^{b}, D_{o}^{A^{b}} \delta \Psi\right]\right) \\
& -(1-\chi) 2\left\langle\Phi^{b} \mid \delta \Psi\right\rangle\left(\frac{\chi_{1}}{r^{2}} \epsilon_{i}{ }^{j}{ }_{k} x^{k} \tau_{j}+Z_{i}^{b}\right), \\
& K_{22}(\delta Z)_{i}=\delta^{k l}\left(\partial_{k}\left(\left[\delta Z_{l}, A_{i}^{b}\right]+\left[A_{l}^{b}, \delta Z_{i}\right]\right)+\left[\delta Z_{k}, F_{l i}^{A^{b}}\right]+\left[A_{k}^{b}, \delta F_{l i}\right]\right) \\
& -\chi\left[\Phi^{b},\left[\delta Z_{i}, \Phi^{b}\right]\right]-\left(\left(\left|\Phi^{b}\right|^{2}-1\right)-\chi_{1}\left|\Phi^{b}\right|^{2}\right) \delta Z_{i},
\end{aligned}
$$

and

$$
\delta F_{l i}=\partial_{l} \delta Z_{i}-\partial_{i} \delta Z_{l}+\left[\delta Z_{l}, A_{i}^{b}\right]+\left[A_{l}^{b}, \delta Z_{i}\right] .
$$

Since $\Delta: \mathcal{U}_{\delta}^{2, p} \rightarrow \mathcal{U}_{2}^{0, p}$ is an isomorphism (see proposition 4.1), it follows from the structure of the (7.10) that $D \Xi_{0}\left(\mathfrak{U}_{b}, \Psi^{b}, Z^{b}\right)$ will be an isomorphism provided that

$$
S:=\left(\begin{array}{cc}
\Delta-2 \frac{w^{2}-1}{r^{2}} & 0 \\
0 & \Delta-1
\end{array}\right)+\left(\begin{array}{cc}
0 & K_{12} \\
K_{21} & K_{22}
\end{array}\right)
$$

is an isomorphism. Let

$$
K=\left(\begin{array}{cc}
0 & K_{12} \\
K_{21} & K_{22}
\end{array}\right)
$$


Then the weighted Rellich-Kondrachov theorems (see lemma 3.8 and lemma 2.1 of [5]), lemma 3.9, theorem 1.2 (iv) of [1], and the inclusion (3.7) shows that map $K: \mathcal{H}_{\delta}^{2, p} \times \mathcal{A}_{\mu}^{2, p} \rightarrow \mathcal{H}_{\delta-2}^{0, p} \times \mathcal{A}_{\mu}^{0, p}$ is compact. As the Index of a operator is preserved under compact perturbations, we get

$$
\operatorname{Index}(S)=0
$$

by propositions 4.2 and 4.5 . Thus if we can establish that $S$ is injective then the proof will be complete.

\section{Lemma 7.2.}

$$
\operatorname{Ker}(S)=0
$$

Proof. We first consider the YMH Lagrangian

$$
L(\Psi, Z)=\int_{\mathbb{R}^{3}} \frac{1}{2} \delta^{i k} \delta^{i j}\left\langle F_{i k}^{A} \mid F_{j l}^{A}\right\rangle+\delta^{i j}\left\langle D_{o}^{A} \Phi \mid D_{o}^{A} \Phi\right\rangle d^{3} x .
$$

where $A=Y+Z$ and $\Phi=\Omega+\Psi$ as above. Since $p>6$ and $-1<\delta<-3 / p$, we get from (6.7), (6.8), theorem 2.1 (i) of [1] and the multiplication lemma 3.3 that the map

$\mathcal{H}_{\delta}^{2, p} \times \mathcal{A}_{\mu}^{2, p} \rightarrow \mathrm{W}_{2 \delta-2}^{0, p}\left(\mathbb{R}^{3}\right) \subset L^{1}\left(\mathbb{R}^{3}\right):(\Psi, Z) \rightarrow \frac{1}{2} \delta^{i k} \delta^{i j}\left\langle F_{i k}^{A} \mid F_{j l}^{A}\right\rangle+\delta^{i j}\left\langle D_{o}^{A} \Phi \mid D_{o}^{A} \Phi\right\rangle$

is analytic. Consequently the Lagrangian (17.17) defines analytic map from $\mathcal{H}_{\delta}^{2, p} \times$ $\mathcal{A}_{\mu}^{2, p}$ to $\mathbb{R}$. Differentiating (7.17) yields

$$
\begin{aligned}
D L(\Psi, Z) \cdot & (\delta \Psi, \delta Z)=\int_{\mathbb{R}^{3}} \delta^{i k} \delta^{i j}\left\langle F_{i k}^{A} \mid \partial_{j} \delta Z_{k}-\partial_{k} \delta Z_{j}+\left[\delta Z_{j}, A_{k}\right]+\left[A_{j}, \delta Z_{k}\right]\right\rangle d^{3} x \\
& +\int_{\mathbb{R}^{3}} 2 \delta^{i j}\left\langle D_{i}^{A} \Phi \mid D_{j}^{A} \delta \Psi+\left[\delta Z_{j}, \Phi\right]\right\rangle d^{3} x \\
= & -\int_{\mathbb{R}^{3}} 2 \delta^{i k} \delta^{j l}\left\langle D_{o}^{A} F_{k j}-\left[\Phi, D_{o}^{A} \Phi\right] \mid \delta Z_{l}\right\rangle+2 \delta^{i j}\left\langle D_{o}^{A} D_{o}^{A} \Phi \mid \delta \Psi\right\rangle d^{3} x
\end{aligned}
$$

where in deriving the last inequality we used integration by parts. A similar calculation shows that the second derivative evaluated on the diagonal is

$$
\begin{aligned}
D^{2} L(\Psi, Z) \cdot((\delta \Psi, \delta Z),(\delta \Psi, \delta Z))= & -2 \int_{\mathbb{R}^{3}} \delta^{i j}\left\langle L_{2}(\Psi, Z) \cdot(\delta \Psi, \delta Z)_{i} \mid \delta Z_{j}\right\rangle \\
& +\left\langle L_{1}(\Psi, Z) \cdot(\delta \Psi, \delta Z) \mid \delta \Psi\right\rangle d^{3} x
\end{aligned}
$$

where

$$
\begin{gathered}
L_{1}(\Psi, Z) \cdot(\delta \Psi, \delta Z)=\delta^{i j}\left(D_{i}^{A}\left(D_{j}^{A} \delta \Psi+\left[\delta Z_{i}, \Phi\right]\right)+\left[\delta Z_{i}, D_{o}^{A} \Phi\right]\right), \\
L_{2}(\Psi, Z) \cdot(\delta \Psi, \delta Z)_{j}=\delta^{i k}\left({ }_{o}^{A}\left(\partial_{k} \delta Z_{j}-\partial_{j} \delta Z_{j}+\left[\delta Z_{k}, A_{j}\right],\left[A_{k}, \delta Z_{j}\right]\right)+\left[\delta Z_{i}, F_{k j}^{A}\right]\right) \\
-\left[\Psi, D_{o}^{A} \Phi\right]-\left[\Phi, D_{o}^{A} \Psi+\left[\delta Z_{j}, \Phi\right]\right] .
\end{gathered}
$$


Let

$$
* F_{k}^{A}=\frac{1}{2} \epsilon^{i j}{ }_{k} F_{i j}^{A}
$$

be the Hodge dual of $F^{A}$. Then the Bianchi identities for $F^{A}$ imply that

$$
\begin{gathered}
\delta^{i j}\left\langle * F_{i}^{A}+{\underset{o}{D}}_{i}^{A} \Phi \mid * F_{j}^{A}+{\underset{o}{D}}_{j}^{A} \Phi\right\rangle d x^{1} \wedge d x^{2} \wedge d x^{3}-d\left(\left\langle\Phi \mid F_{i j}^{A}\right\rangle d x^{i} \wedge d x^{j}\right)= \\
\left(\frac{1}{2} \delta^{i k} \delta^{i j}\left\langle F_{i k}^{A} \mid F_{j l}^{A}\right\rangle+\delta^{i j}\left\langle\underset{o}{D_{i}^{A}} \Phi \mid D_{o}^{A} \Phi\right\rangle\right) d x^{1} \wedge d x^{2} \wedge d x^{3} .
\end{gathered}
$$

Therefore the Lagrangian (7.17) can be written as

$$
L(\Psi, Z)=\int_{\mathbb{R}^{3}} \delta^{i j}\left\langle * F_{i}^{A}+D_{o}^{A} \Phi \mid * F_{j}^{A}+D_{o}^{A} \Phi\right\rangle d^{3} x-\int_{\mathbb{R}^{3}} d\left(\left\langle\Phi \mid F_{i j}^{A}\right\rangle d x^{i} \wedge d x^{j}\right) .
$$

But for $(\Psi, Z) \in \mathcal{H}_{0}^{\infty} \times \mathcal{A}_{0}^{\infty}$ we have we have that

$$
\begin{gathered}
\int_{\mathbb{R}^{3}} d\left(\left\langle\Phi \mid F_{i j}^{A}\right\rangle d x^{i} \wedge d x^{j}\right)=\int_{\mathbb{R}^{3}} d\left(\left\langle\Omega \mid F_{i j}^{Y}\right\rangle d x^{i} \wedge d x^{j}\right) \\
+\int_{\mathbb{R}^{3}} d\left(\left\langle\Omega \mid F_{i j}^{Z}+\left[Z_{i}, Y_{j}\right]+\left[Y_{i}, Z_{j}\right]\right\rangle+\left\langle\Psi \mid F_{i j}^{Y}+F_{i j}^{Z}+\left[Z_{i}, Y_{j}\right]+\left[Y_{i}, Z_{j}\right]\right\rangle d x^{i} \wedge d x^{j}\right) \\
=\lim _{R \rightarrow \infty} \int_{\partial B_{R}(0)}\left\langle\Omega \mid F_{i j}^{Y}\right\rangle d x^{i} \wedge d x^{j}=4 \pi
\end{gathered}
$$

where we have used Stokes' theorem to convert to a surface integral. Using the weighted Sobolev inequalities (see theorem 3.7 and theorem 1.2 of [1]), it follows from the density of $\mathcal{H}_{0}^{\infty} \times \mathcal{A}_{0}^{\infty}$ that

$$
\int_{\mathbb{R}^{3}} d\left(\left\langle\Phi \mid F_{i j}^{A}\right\rangle d x^{i} \wedge d x^{j}\right)=4 \pi \text { for all }(\Psi, Z) \in \mathcal{H}_{\delta}^{2, p} \times \mathcal{A}_{\mu}^{2, p} .
$$

Thus we have the alternate form for the Lagrangian

$$
L(\Psi, Z)=\int_{\mathbb{R}^{3}} \delta^{i j}\left\langle * F_{i}^{A}+D_{o}^{A} \Phi \mid * F_{j}^{A}+D_{o}^{A} \Phi\right\rangle d^{3} x-4 \pi .
$$

This way of expressing the Yang-Mills-Higgs Lagrangian is well known and leads to Bogomol'nyi first order equations. Differentiating the above Lagrangian twice and using integration by parts yields

$$
\begin{gathered}
D^{2} L(\Psi, Z) \cdot((\delta \Psi, \delta Z),(\delta \Psi, \delta Z))=\int_{\mathbb{R}^{3}} 2 \delta^{i j}\left\langle * F_{i}^{A}+D_{o}^{A} \Phi \mid M_{1}(\delta \Psi, \delta Z)_{j}\right\rangle+ \\
\left.\left.2 \delta^{i j}\left\langle M_{2}(\Psi, Z) \cdot(\delta \Psi, \delta Z)_{i}\right| M_{2}(\Psi, Z) \cdot(\delta \Psi, \delta Z)_{j}\right)\right\rangle d^{3} x
\end{gathered}
$$

where

$$
\begin{aligned}
M_{1}(\delta \Psi, \delta Z)_{k} & =\epsilon_{k}^{i j}\left[\delta Z_{i}, \delta Z_{j}\right]+2\left[\delta Z_{k}, \delta \Psi\right] \\
M_{2}(\Psi, Z) \cdot(\delta \Psi, \delta Z)_{k} & =\frac{1}{2} \epsilon^{i j}{ }_{k}\left(\partial_{i} \delta Z_{j}-\partial_{j} \delta Z_{i}+\left[\delta Z_{i}, A_{j}\right]+\left[A_{i}, \delta Z_{j}\right]\right) \\
& +\left(D_{k}^{A} \delta \Psi+\left[\delta Z_{k}, \Phi\right]\right) .
\end{aligned}
$$


Now suppose that $(\delta \Psi, \delta Z) \in \mathcal{H}_{\delta}^{2, p} \times \mathcal{A}_{\mu}^{2, p}$ satisfies $S(\delta \Psi, \delta Z)=0$. Since $S$ is an elliptic operator with smooth coefficients, elliptic regularity implies that $\delta \Psi$ and $\delta Z$ are $C^{\infty}$. Then using (5.2) and (7.9) $\delta \Psi$ and $\delta Z$ satisfy

$$
L_{1}\left(\Psi^{b}, Z^{b}\right) \cdot(\delta \Psi, \delta Z)=0 \quad \text { and } \quad L_{2}\left(\Psi^{b}, Z^{b}\right) \cdot(\delta \Psi, \delta Z)=0 .
$$

Also, we note that $\Phi^{b}$ and $A^{b}$ satisfy the Bogomol'nyi equations

$$
* F_{j}^{A^{b}}+D_{o}^{A^{b}} \Phi^{b}=0 \quad \Longleftrightarrow \quad w^{\prime}+w \phi=0, \quad r^{2} \phi^{\prime}+w^{2}-1=0 .
$$

So we get by (7.18) and (7.22) that

$$
\frac{1}{2} \epsilon^{i j}\left(\partial_{i} \delta Z_{j}-\partial_{j} \delta Z_{i}+\left[\delta Z_{i}, A_{j}^{b}\right]+\left[A_{i}^{b}, \delta Z_{j}\right]\right)+\left(D_{k}^{A^{b}} \delta \Psi+\left[\delta Z_{k}, \Phi^{b}\right]\right)=0 .
$$

Letting $\delta \Psi=\psi(r) r^{-1} x^{k} \tau_{k}$ and $\delta Z_{i}=z(r) r^{-2} \epsilon_{i}^{j} x^{k} \tau_{j}$, we can write (7.25) as

$$
z^{\prime}+\phi z+w \psi=0 \text { and } r^{2} \psi^{\prime}+2 w z=0,
$$

where $w(r)$ and $\phi(r)$ are given by (7.6). Differentiating $1 / w$ times the second equation and then using the two equations to eliminate $z$ and $z^{\prime}$ yields

$$
\left(r^{2} \psi^{\prime}\right)^{\prime}+2 \phi r^{2} \psi^{\prime}-2 w^{2} \psi=0 .
$$

Since $\delta \Psi \in C^{\infty} \cap \mathcal{A}_{\mu}^{2, p}(-1<\delta<0)$ we get that $\psi(r)=\mathrm{O}(r)$ as $r \rightarrow 0$ and that $\psi(r)=o\left(r^{\delta}\right)$ as $r \rightarrow \infty$ by theorem 1.2 of [1]. Since $w>0$ on $[0, \infty)$ the only solution satisfying the differential equation (17.27) and the asymptotic conditions is the trivial solution $\psi=0$. But $\psi=0$ implies that $z=0$ and thus $\delta \Psi=0$ and $\delta Z=0$. This establishes that $\operatorname{Ker}(S)$ is trivial.

We can now solve the reduced/modified EYMH equations.

Theorem 7.3. Suppose $p>6,-1<\delta<-3 / p$ and $-1<\mu<0$. Then there exists $a \Lambda>0$ and an analytic map

$$
(-\Lambda, \Lambda) \longrightarrow \mathfrak{U}_{\delta}^{2, p} \times \mathcal{H}_{\delta}^{2, p} \times \mathcal{A}_{\mu}^{2, p}: \lambda \longmapsto(\mathfrak{U}(\lambda), \Psi(\lambda), Z(\lambda))
$$

such that $(\mathfrak{U}(0), \Psi(0), Z(0))=\left(\mathfrak{U}_{b}, \Psi^{b}, Z^{b}\right)$ and $\Xi(\lambda, \mathfrak{U}(\lambda), \Psi(\lambda), Z(\lambda))=0$ for all $\lambda \in(-\Lambda, \Lambda)$.

Proof. Propositions 6.5 and 7.1 and the results of section 7.1 allow us to apply the analytic version of the implicit function theorem (see [6] theorem 15.3) to reach the desired conclusion. 


\section{Existence}

We have so far only found a solution to the reduced/modified EYMH equations (2.18), (5.4), and (5.5). However, we will now show that the solution obtained in theorem 7.3 is also a solution to the EYMH equations (2.25)-(2.26).

Proposition 8.1. Suppose $p>6,-1<\delta<-3 / p$, and $-1<\mu<0$. Let

$$
(-\Lambda, \Lambda) \longrightarrow \mathfrak{U}_{\delta}^{2, p} \times \mathcal{H}_{\delta}^{2, p} \times \mathcal{A}_{\mu}^{2, p}: \lambda \longmapsto(\mathfrak{U}(\lambda), \Psi(\lambda), Z(\lambda))
$$

be the map from theorem 7.3. Then there exists a $\Lambda^{*} \in(0, \Lambda]$ such that for every $\lambda \in\left(-\Lambda^{*}, \Lambda^{*}\right),(\mathfrak{U}(\lambda), \Phi(\lambda)=\Omega+\Psi(\lambda), A(\lambda)=Y+Z(\lambda))$ solves the $Y M H$ equations (2.25) -(2.26) and $(\Psi(\lambda), Z(\lambda)) \in \mathcal{U}_{\delta}^{2, p} \cap C^{1} \times \mathcal{H}_{\delta}^{2, p} \cap C^{2} \times \mathcal{A}_{\mu}^{2, p} \cap C^{2}$.

Proof. Fix $R>0$. Then for each $\lambda \in(-\Lambda, \Lambda), \mathfrak{U}(\lambda) \in \mathrm{W}^{2, p}\left(B_{R}(0), \mathbb{S}^{3}\right), \Psi \in$ $\mathrm{W}^{2, p}\left(B_{R}(0), \mathfrak{s u}(2)\right)$, and $Z(\lambda) \in \mathrm{W}^{2, p}\left(B_{R}(0), \mathfrak{s u}(2)^{3}\right)$. To reduce notation we will often write $\mathfrak{U}, \Psi$, and $Z$ instead of $\mathfrak{U}(\lambda), \Psi(\lambda)$, and $Z(\lambda)$. Since $Y$ and $\Omega$ are $C^{\infty}$ it follows from (6.14)-(6.15) and the Sobolev inequalities that

$$
\overline{\mathfrak{g}}^{i j} \partial_{i j}^{2} \Psi=f \quad \text { and } \quad Q^{i j k}{ }_{l} \partial_{i j}^{2} Z_{k}=h_{l}
$$

where $f, h_{l} \in \mathrm{W}^{1, p}\left(B_{R}(0), \mathfrak{s u}(2)\right) \subset C^{0,1-3 / p}\left(B_{R}(0), \mathfrak{s u}(2)\right)$ and

$$
\begin{gathered}
\overline{\mathfrak{g}}^{i j}=\delta^{i j}+4 \lambda^{2} \mathfrak{U}^{i j}, \\
Q^{i k}=\left(Q^{i k l}{ }_{j}\right):=\left(\left(\delta^{i k}+4 \lambda^{2} \mathfrak{U}^{i k}\right) \delta_{j}^{l}-4 \lambda^{2} \mathfrak{U}^{l k} \delta_{j}^{i}\right)
\end{gathered}
$$

By the weighted Sobolev inequality, [1] theorem $1.2(\mathrm{v})$, the embedding $\mathrm{W}_{\delta}^{1, p}\left(\mathbb{R}^{3}, \mathbb{S}^{3}\right) \rightarrow$ $\mathrm{C}_{\delta}^{0,1-3 / p}\left(\mathbb{R}^{3}, \mathbb{S}^{3}\right)$ is continuous and hence the map $(-\Lambda, \Lambda) \rightarrow \mathrm{C}_{\delta}^{0,1-3 / p}\left(\mathbb{R}^{3}, \mathbb{S}^{3}\right)$ : $\lambda \mapsto U(\lambda)$ is continuous. Therefore, there exists a $\Lambda^{*} \in(0, \Lambda)$ such that the operators $\overline{\mathfrak{g}}^{i j} \partial_{i j}^{2}$ and $Q^{i j} \partial_{i j}^{2}$ are uniformly elliptic with with coefficients in $\mathrm{C}_{\delta}^{0,1-3 / p}\left(\mathbb{R}^{3}\right)$ for all $\lambda \in\left[-\Lambda^{*}, \Lambda^{*}\right]$. By elliptic regularity, $\Psi, Z_{k} \in C^{2}\left(B_{R}(0), \mathfrak{s u}(2)\right)$. As $\Lambda^{*}$ is independent of $R$, we get that $\Psi(\lambda), Z_{k}(\lambda) \in C^{2}\left(\mathbb{R}^{3}, \mathfrak{s u}(2)\right)$ for all $\lambda \in\left(-\Lambda^{*}, \Lambda^{*}\right)$.

For $\lambda>0$ we can, using (2.6), recover the metric $g_{\alpha \beta}$ from $\mathfrak{U}_{\alpha \beta}$. Since $\mathfrak{U} \in$ $\mathrm{W}_{\delta}^{2, p}\left(\mathbb{R}^{3}, \mathbb{S}\right)$, we have by theorem $1.2(\mathrm{v})$ of [1] that $\mathfrak{U}^{\alpha \beta} \in \mathrm{C}_{\delta}^{0,1-3 / p}\left(\mathbb{R}^{3}\right)$ and $\partial_{k} \mathfrak{U}^{\alpha \beta} \in \mathrm{C}_{\delta-1}^{0,1-3 / p}\left(\mathbb{R}^{3}\right)$. Therefore, in spherical coordinates the metric becomes

$$
g_{\alpha \beta} d x^{\alpha} d x^{\beta}=-S(r) N(r) d t^{2}+\frac{1}{N(r)} d r^{2}+R(r)^{2}\left(d \theta^{2}+\sin ^{2} \theta d \phi^{2}\right),
$$

where $N, S$, and $R$ are in $C^{1}((0, \infty))$. But then a straightforward calculation shows that for all $r \in(0, \infty)$

$$
\begin{aligned}
& \left(1-\chi_{3}\right) g^{\alpha \beta} \mathrm{D}_{\alpha}^{Y} F_{\beta \nu}^{Y} d x^{\nu}= \\
& \quad\left(1-\chi_{3}\right)\left(\frac{1}{S}\left(N S \chi_{1}^{\prime}\right)^{\prime}-\frac{\left(\chi_{1}^{2}-1\right) \chi_{1}}{R^{2}}\right)\left(-\sin \phi \tau_{1}+\cos \phi \tau_{2}\right) d \theta \\
& \quad+\left(\chi_{3}-1\right)\left(\frac{1}{S}\left(N S \chi_{1}^{\prime}\right)^{\prime}-\frac{\left(\chi_{1}^{2}-1\right) \chi_{1}}{R^{2}}\right)\left(\tau_{3}-\cot \theta\left(\sin \phi \tau_{2}+\cos \phi \tau_{1}\right)\right) d \phi=0 .
\end{aligned}
$$


where $(\cdot)^{\prime}=\frac{d}{d r}(\cdot)$. Using this result and the identity (5.2) which is valid for $C^{1}$ static spherically symmetric fields, it is clear that $(\mathfrak{U}, \Psi, Z)$ satisfy the $\mathrm{YMH}$ equations.

To complete the existence proof, we now use the following result of Heilig.

Proposition 8.2. [proposition 6.1, [9]] Suppose $-1<\delta<0, p>3$, and $\Lambda>0$. Furthermore, suppose

$$
T:[0, \Lambda] \rightarrow \mathrm{W}_{\delta-2}^{0, p}\left(\mathbb{R}^{3}, \mathbb{S}^{3}\right) \cap \mathrm{C}^{1}\left(\mathbb{R}^{3}, \mathbb{S}^{3}\right): \lambda \mapsto\left(T_{\lambda}^{\alpha \beta}\right)
$$

and

$$
\mathfrak{U}:[0, \Lambda] \rightarrow \mathrm{W}_{\delta}^{2, p}\left(\mathbb{R}^{3}, \mathbb{S}^{3}\right): \lambda \mapsto\left(\mathfrak{U}_{\lambda}^{\alpha \beta}\right)
$$

are two continuous maps such that for every $\lambda \in[0, \Lambda]:\left(\lambda, \mathfrak{U}_{\lambda}^{\alpha \beta}, T_{\lambda}^{\alpha \beta}\right)$ is a solution to the reduced field equations 2.17, $\nabla_{\beta} T_{\lambda}^{\alpha \beta}=0$, and $\partial_{\gamma} T_{\lambda}^{\alpha \beta} \in B_{\mathrm{W}_{\delta-2}^{0, p}\left(\mathbb{R}^{3}\right)}(0, R)$ for some $R>0$ independent of $\lambda$ and $\alpha, \beta, \gamma$. Then there exists a constant $\hat{\Lambda} \in(0, \Lambda]$ such that $\partial_{\alpha} \mathfrak{U}_{\lambda}^{\alpha \beta}=0$ for all $\lambda \in[0, \hat{\Lambda}]$.

Theorem 8.3. Suppose $p>6,-1<\delta<-3 / p$, and $-1<\mu<0$. Let

$$
(-\Lambda, \Lambda) \longrightarrow \mathfrak{U}_{\delta}^{2, p} \times \mathcal{H}_{\delta}^{2, p} \times \mathcal{A}_{\mu}^{2, p}: \lambda \longmapsto(\mathfrak{U}(\lambda), \Psi(\lambda), Z(\lambda))
$$

be the map from theorem 7.3. Then there exists a $\Lambda^{*} \in(0, \Lambda]$ such that for every $\lambda \in\left(-\Lambda^{*}, \Lambda^{*}\right),(\mathfrak{U}(\lambda), \Phi(\lambda)=\Omega+\Psi(\lambda), A(\lambda)=Y+Z(\lambda))$ solves the $E Y M H$ equations (2.17)-(2.18) and (2.25)-(2.26). Moreover, $(\mathfrak{U}(\lambda), \Psi(\lambda), Z(\lambda)) \in \mathcal{U}_{\delta}^{2, p} \cap$ $C^{2} \times \mathcal{H}_{\delta}^{2, p} \cap C^{2} \times \mathcal{A}_{\mu}^{2, p} \cap C^{2}$ for all $\lambda \in\left(-\Lambda^{*}, \Lambda^{*}\right)$.

Proof. From proposition 8.1 we know that there exist a $\Lambda^{*} \in(0, \Lambda]$ such that $(\mathfrak{U}(\lambda), \Phi(\lambda)=\Omega+\Psi(\lambda), A(\lambda)=Y+Z(\lambda))$ solves the YMH equations (2.25)-(2.26). and $\mathfrak{U}(\lambda) \in C^{1}\left(\mathbb{R}^{3}, \mathbb{S}^{3}\right), \Psi(\lambda), A_{k}(\lambda) \in C^{2}\left(\mathbb{R}^{3}, \mathfrak{s u}(2)\right)$ for all $\lambda \in\left(-\Lambda^{*}, \Lambda\right)$. It can then be checked that the YMH equations imply that $\nabla_{\alpha} T^{\alpha \beta}=0$ is automatically satisfied. Therefore, the harmonic equation

$$
\partial_{\alpha} \mathfrak{U}^{\alpha \beta}=0
$$

is satisfied for all $\lambda \in\left(-\Lambda^{*}, \Lambda\right)$ by propositions 6.3 and 8.2. So we have shown that $(\mathfrak{U}(\lambda), \Phi(\lambda)=\Omega+\Psi(\lambda), A(\lambda)=Y+Z(\lambda))$ satisfies the EYMH equations (2.17)-(2.18) and (2.25)-(2.26) for all $\lambda \in\left(-\Lambda^{*}, \Lambda\right)$. To complete the the proof we use (8.1) to write the reduced equations (2.18) as

$$
\overline{\mathfrak{g}}^{i j} \partial_{x^{i} x^{j}}^{2} \mathfrak{U}^{\alpha \beta}=H^{\alpha \beta}
$$

where $H^{\alpha \beta}=-A^{\alpha \beta}-B^{\alpha \beta}-C^{\alpha \beta}+4 \pi G|\mathfrak{d}| T^{\alpha \beta}$. As in proposition 8.1, it can be shown that there exist a $\hat{\Lambda}>0$ such that for all $\lambda \in(0, \hat{\Lambda})$ and $R>0$ that $H^{\alpha \beta} \in \mathrm{C}^{0,1-3 / p}\left(\mathrm{~B}_{R}\right)$ and the operator $\overline{\mathfrak{g}}^{i j} \partial_{i j}^{2}$ is uniformly elliptic with coefficients in $C_{\delta}^{0,1-3 / p}\left(\mathbb{R}^{3}\right)$. Therefore we conclude via elliptic regularity that $\mathfrak{U}^{\alpha \beta} \in \mathrm{C}^{2}$. 
As the Newtonian solutions (7.7) and (7.8) are $C^{\infty}$, we do not have to restrict the differentiability to $k=2$. All the same arguments go through for $k \geq 2$. Then using the weighted Sobolev inequalities we get the following result:

Corollary 8.4. Suppose $-1<\delta<0$, and $-1<\mu<0,0<\alpha<1$. Then for any integer $k \geq 2$ there exist a constant $\Lambda>0$ and an analytic map

$(-\Lambda, \Lambda) \longrightarrow C_{\delta}^{k, \alpha}\left(\mathbb{R}^{3}, \mathbb{S}\right) \times C_{\delta}^{k, \alpha}\left(\mathbb{R}^{3}, \mathfrak{s u}(2)\right) \times \mathcal{C}_{\mu}^{k, \alpha}\left(\mathbb{R}^{3}, \mathfrak{s u}(2)^{3}\right): \lambda \longmapsto(\mathfrak{U}(\lambda), \Psi(\lambda), Z(\lambda))$

such that for every $\lambda \in(-\Lambda, \Lambda),(\mathfrak{U}(\lambda), \Phi(\lambda)=\Omega+\Psi(\lambda), A(\lambda)=Y+Z(\lambda))$ solves the EYMH equations (2.17)-(2.18) and $\left(\mathfrak{U}(0)=\mathfrak{U}_{b}, \Phi(0)=\Phi^{b}, A(0)=A^{b}\right)$.

Acknowledgements. This work was partially supported by the ARC grant A00105048 at the University of Canberra and by the NSERC grants A8059 and 203614 at the University of Alberta.

\section{References}

[1] R. Bartnik, The Mass of an Asymptotically Flat Manifold, Comm. Pure Appl. Math. 39 (1986), 661-693.

[2] R. Bartnik, The spherically symmetric Einstein Yang-Mills equations, Relativity Today (Z. Perjés, ed.), 1989, Tihany, Nova Science Pub., Commack NY, 1992, 221-240.

[3] R. Beig and B. Schmidt, Time-Independent Gravitational Fields, Lect. Notes Phys. 540 (2000), 325-372.

[4] P. Breitenlohner and P. Forgaács and D. Maison, Gravitating monopole solutions, Nucl. Phys. B 383 (1992), 357-376.

[5] Y. Choquet-Bruhat and D. Christodoulou, Elliptic systems in $H_{s, \delta}$ spaces on manifolds which are euclidean at infinity, Acta. Math. 146 (1981), 129-150.

[6] K. Deimling, Nonlinear functional analysis, Springer-Verlag, Berlin, 1985.

[7] M. Giaquinta, Introduction to Regularity Theory for Nonlinear Elliptic Systems, Birkhäuser, Berlin, 1993.

[8] D. Gilbarg and N. S. Trudinger, Elliptic Partial Differential Equations of Second order, $2^{\text {nd }}$ ed., Revised $3^{\text {rd }}$ printing, Springer, Berlin, 1998.

[9] U. Heilig, Existence of Rotating Stars in General Relativity, Comm. Math. Phys. 166 (1995), 457-493.

[10] A. Jaffe and C. Taubes, Vortices and monopoles: structure of static gauge theories, Birkhäuser, Boston, 1980. 
[11] H.P. Künzle and C. Duval, Relativistic and nonrelativistic physical theories on five-dimensional space-time, Semantical aspects of spacetime theories (U. Majer and H.-J. Schmidt, eds.), BI-Wissenschaftsverlag, Mannheim 1994, 113129.

[12] E. H. Lieb and M. Loss, Analysis, $2^{\text {nd }}$ ed., AMS, Providence, 2001.

[13] M. Lottermoser, A convergent Post-Newtonian Approximation for the Constraint equations in General Relativity, Ann. Inst. Heri Poincar'e 57 (1992), 279-317.

[14] M.K. Prasad and C.M. Sommerfield, Exact classical solutions for the t'Hooft monopole and the Julia-Zee dyon, Phys. Rev. Lett. 35 (1975), 760-762. 